Supporting Information

\title{
Waste Polypropylene Plastic Recycling Towards Climate Change Mitigation and Circular Economy: Energy, Environmental and Technoeconomic Perspectives
}

\author{
Raaj R. Bora ${ }^{1}$, Ralph Wang ${ }^{2}$, Fengqi You ${ }^{1 *}$ \\ ${ }^{1}$ Robert Frederick Smith School of Chemical and Biomolecular Engineering, Cornell University, Ithaca, \\ New York 14853, USA \\ ${ }^{2}$ College of Engineering, Cornell University, Ithaca, New York 14853, USA
}

This file contains: 31 pages, 4 sections, 24 tables and 6 figures.

* Corresponding author. E-mail: fengqi.you@cornell.edu 


\section{$\underline{\text { List of Sections }}$}

S1. Existing literature on modeling

S1.1 Gasification

S1.2 Fast pyrolysis

S2. Parameters and calculations for the simulations, LCA, and TEA

S2.1 Simulation parameters and results

S2.2 LCA and TEA parameters and results

S3. Pyrolysis of polypropylene mixtures

S3.1 Polypropylene interactions with polystyrene in pyrolysis

S3.2 Polypropylene interactions with polyethylene in pyrolysis

S3.3 Polypropylene interactions with polyvinyl chloride in pyrolysis

S3.4 Polypropylene interactions with polyethylene terephthalate in pyrolysis

S4. Gasification of polypropylene mixtures

S4.1 Polypropylene interactions with polyethylene in gasification

S4.2 Polypropylene interactions with polystyrene in gasification

S4.3 Polypropylene interactions with polyethylene terephthalate in gasification

S4.4 Polypropylene interactions with polyvinyl chloride in gasification

\section{List of Tables}

- Table S1. Fast Pyrolysis product distribution with changing temperature.

- Table S2. Composition of the heavy hydrocarbon fraction from fast pyrolysis.

- Table S3. Gas composition from the fast pyrolysis (excluding nitrogen).

- Table S4. Composition of the light hydrocarbon fraction from fast pyrolysis.

- Table S5. Composition of the aromatics fraction from fast pyrolysis.

- Table S6. Characterization of emissions reported from landfills for municipal solid waste.

- Table S7. Gas composition from the gasification process.

- Table S8. Characterization of emissions reported from incinerators for municipal solid waste.

- Table S9. Names, operating parameters and modeling units used for the important components in the Aspen Plus simulations.

- Table S10. Factors used for calculation of the total project investment and capital investment values.

- Table S11. Annualized cost distribution

- Table S12. NPV calculation for fast pyrolysis.

- Table S13. Sensitivity analysis parameters for fast pyrolysis.

- Table S14. Sensitivity analysis parameters for gasification.

- Table S15. ReCiPe results for fast pyrolysis of 1 ton of waste PP. 
- Table S16. ReCiPe results for gasification of 1 ton of waste PP.

- Table S17. ReCiPe results for landfilling of 1 ton of waste PP.

- Table S18. ReCiPe results for incineration of 1 ton of waste PP.

- Table S19. ILCD, 2.0 results for fast pyrolysis of 1 ton of waste PP.

- Table S20. ILCD, 2.0 results for gasification of 1 ton of waste PP.

- Table S21. ILCD, 2.0 results for landfilling of 1 ton of waste PP.

- Table S22. ILCD, 2.0 results for incineration of 1 ton of waste PP.

- Table S23. Characterization factor units used for the ReCiPe method.

- Table S24. Characterization factor units for the ILCD 2.0 method.

\section{$\underline{\text { List of Figures }}$}

- Figure S1. The annual production costs for the systems analyzed. Both incineration and landfilling are represented by cases with and without the capital costs (CC).

- Figure S2. Comparison of the NPV for the gasification and fast pyrolysis pathways.

- Figure S3. ILCD contribution analysis for fast pyrolysis.

- Figure S4. ILCD contribution analysis for gasification.

- Figure S5. ILCD contribution analysis for landfilling.

- Figure S6. ILCD contribution analysis for incineration. 


\section{S1. Existing literature on modeling}

There are existing investigations and experiments to determine the kinetic and thermodynamic data for the gasification and fast pyrolysis of PP wastes. Certain studies consider an isolated PP stream whereas others combine PP with one or more different waste plastic types to analyze their combined effects of the feed on the corresponding products.

\section{S1.1 Gasification}

It is observed that the conversion rates of polypropylene into syngas are quite high, both with and without catalysts, with the main challenge being tar formation. For instance, Wu and Williams reported high mass conversions of $96 \%$ and $99 \%$ in their two-stage pyrolysis-steam gasification system using $\mathrm{Ni}-\mathrm{Al}$ and $\mathrm{Ni}-\mathrm{Mg}$-Al catalysts, respectively. ${ }^{1}$ Xiao et al reported similarly high mass yields between $76 \%$ and $99 \%$ for the non-catalytic air gasification of polypropylene. Xiao et al also investigated the effects of the equivalence ratio (ER) and temperature on the products. As ER increased, more combustion occurred, increasing the temperature. These effects increased the conversion of PP into products, but the products had lower energy densities. ${ }^{2}$

The primary challenge in plastics gasification is the reduction of product tar content. Tar production in gasification often exceeds the acceptable limits of syngas applications, so steps need to be taken to either suppress tar formation or to precipitate tars from the product gas. ${ }^{3}$ Xiao et al reported that tar production is negatively correlated with the ratio of the oxidizer used to the oxidizer needed for complete combustion, also known as ER. ${ }^{2}$ At the same time, increasing ER is also correlated with decreased energy content of the output gas. Some authors have investigated the effects of reactor bed materials on tar production. Arena et al reported that application of a calcified olivine reactor bed can dramatically reduce tar production, and when the reactor is heated to $850-900^{\circ} \mathrm{C}$, the tar content in the product gas is nearly undetectable. ${ }^{4-5}$ Another approach to producing syngas with low tar content is two-stage pyrolysis-gasification, where the feed is first pyrolyzed, then the hydrocarbon products are gasified with catalysts to remove the tar content and improve the hydrogen yield. ${ }^{1,6}$ 


\section{S1.2 Fast pyrolysis}

Extensive literature, including thermogravimetric analyses (TGA), product analyses, and mechanistic modelling, is present for the pyrolysis of both polypropylene (PP) and plastic mixtures. ${ }^{7}$ TGA of virgin PP shows that thermal degradation begins at $400^{\circ} \mathrm{C}$, reaches its peak between 450 and $475^{\circ} \mathrm{C}$, and finishes around $500^{\circ} \mathrm{C}$, suggesting an optimal, uncatalyzed pyrolysis temperature between $450^{\circ} \mathrm{C}$ and $500^{\circ} .{ }^{8}$ Ciliz et al extended this work with TGA of waste PP, finding that waste PP degrades at temperatures $20^{\circ} \mathrm{C}$ cooler than that of virgin PP. ${ }^{9}$ This difference in degradation temperature implies that waste PP degrades more readily than its virgin counterpart.

All of the literature reviewed reported a significant oil fraction and a relatively small gas fraction in the product distribution of uncatalyzed PP pyrolysis, but the solid fraction was not consistent across studies. On one extreme, Williams and Slaney reported a mass yield of 95\% oil and 5\% gas. On the other hand, Predel and Kaminsky reported a mass yield of 57.5\% waxes, 35.4\% oil, and 6.9\% gas. ${ }^{10-11}$ Kruse et al modeled the reaction kinetics of polypropylene decomposition between 350 and $420^{\circ} \mathrm{C}$, finding good agreement between the modeled low molecular weight product distribution and experimental results. ${ }^{12}$ Other authors have investigated catalytic pyrolysis of PP. Achilias et al pyrolyzed PP at $450^{\circ} \mathrm{C}$ using an acid FCC catalyst and reported a mass yield of $67.3 \%$ liquid, $26.5 \%$ residues, and $6.2 \%$ gas product. ${ }^{13}$ Kassargy et al investigated pyrolysis of PP, PE, and their mixtures over USY zeolite catalyst, and reported a mass yield of about 78\% liquid, 20\% gas, and very little solid production. ${ }^{14}$

\section{S2. Parameters and calculations for the simulations, LCA, and TEA}

\section{S2.1 Simulation parameters and results}

The operating conditions for the simulations of the chemical recycling technologies were based on multiple published articles and government reports. ${ }^{15-22}$ After the selection of initial operating parameters, an effort was made to update certain operating parameters continuously to ensure the best performance of the systems as shown in the following tables. 
Table S1. Fast Pyrolysis product distribution with changing temperature.

\begin{tabular}{llrr}
\hline Temperature & $\begin{array}{l}\text { Gases } \\
\text { (wt \%) }\end{array}$ & $\begin{array}{l}\text { Oil/tar } \\
\text { (wt \%) }\end{array}$ & \multicolumn{2}{l}{$\begin{array}{l}\text { Solid } \\
\text { (Ash) } \\
\text { (wt \%) }\end{array}$} \\
\hline 300 & 12.28 & 85.77 & 1.95 \\
400 & 13.4 & 85.79 & 1.81 \\
500 & 15.65 & 82.75 & 1.6 \\
600 & 16.31 & 82.65 & 1.04 \\
\hline
\end{tabular}

Table S2. Composition of the heavy hydrocarbon fraction from fast pyrolysis.

\begin{tabular}{lc}
\hline Compound & $\begin{array}{c}\text { Heavy HC fraction } \\
\text { stream (kg/hour) }\end{array}$ \\
\hline N-decane & 56.43 \\
N-undecane & 77.92 \\
N-dodecene & 78.28 \\
N-tridecane & 88.98 \\
N-tetradecane & 90.34 \\
N-pentadecane & 75.09 \\
N-hexadecane & 69.31 \\
N-heptadecane & 60.76 \\
N-octadecane & 53.59 \\
N-nonadecane & 50.08 \\
\hline
\end{tabular}

Table S3. Gas composition from the fast pyrolysis (excluding nitrogen)

\begin{tabular}{ll}
\hline Gas component & $\mathrm{Wt}(\%)$ \\
\hline Hydrogen & 0.13 \\
Methane & 2.56 \\
Ethane & 4.27 \\
Ethylene & 9.6 \\
Propane & 3.95 \\
Propylene & 10.35 \\
\hline
\end{tabular}


Table S4. Composition of the light hydrocarbon fraction from fast pyrolysis.

\begin{tabular}{lc}
\hline Compound & Light HC fraction Stream (kg/hour) \\
\hline Propane & 643.54 \\
N-butane & 5798.49 \\
N-pentane & 2467.98 \\
N-hexane & 933.76 \\
N-heptane & 324.87 \\
N-octane & 173.19 \\
N-nonane & 123.33 \\
N-decane & 156.09 \\
N-undecane & 84.87 \\
N-dodecene & 35.56 \\
N-tridecane & 23.19 \\
\hline
\end{tabular}

Table S5. Composition of the aromatics fraction from fast pyrolysis.

\begin{tabular}{lc}
\hline Compound & $\begin{array}{c}\text { Aromatics Stream } \\
\text { (kg/hour) }\end{array}$ \\
\hline Benzene & 312.44 \\
Toluene & 97.56 \\
Styrene & 145.34 \\
1-heptene & 7.99 \\
1-octene & 11.27 \\
o-xylene & 22.12 \\
1-nonene & 38.76 \\
Naphthalene & 12.04 \\
\hline
\end{tabular}

Table S6. Characterization of emissions reported from landfills for municipal solid waste. ${ }^{23}$

\begin{tabular}{lrl}
\hline Landfill gas & Parameters & Units \\
\hline Methane content & 55 & $\%$ \\
CO2 content & 45 & $\%$ \\
Energy content & 19,730 & $\mathrm{~kJ} / \mathrm{m} 3$ \\
$\begin{array}{l}\text { Gas collection } \\
\text { efficiency }\end{array}$ & 75 & $\%$
\end{tabular}


Leachate characteristics

\begin{tabular}{lll} 
BOD & $2.79 \mathrm{E}+02$ & $\mathrm{mg} / \mathrm{L}$ \\
COD & $9.67 \mathrm{E}+02$ & $\mathrm{mg} / \mathrm{L}$ \\
TSS & $1.91 \mathrm{E}+02$ & $\mathrm{mg} / \mathrm{L}$ \\
NH3 & $2.47 \mathrm{E}+02$ & $\mathrm{mg} / \mathrm{L}$ \\
Total nitrogen & $3.52 \mathrm{E}+02$ & $\mu \mathrm{g} / \mathrm{L}$ \\
Phosphorus & $3.00 \mathrm{E}+00$ & $\mathrm{mg} / \mathrm{L}$ \\
\hline
\end{tabular}

Table S7. Gas composition from the gasification process.

\begin{tabular}{ll}
\hline Gas component & Wt (\%) \\
\hline Hydrogen & 6.08 \\
Carbon Monoxide & 3.12 \\
Methane & 17.32 \\
Ethylene & 73.64 \\
\hline
\end{tabular}

Table S8. Characterization of emissions reported from incinerators for municipal solid waste. ${ }^{23}$

\begin{tabular}{lrl}
\hline Pollutants & Parameters & Units \\
Arsenic & $2.12 \mathrm{E}-03$ & $\mathrm{~kg} / \mathrm{Mg}$ \\
Cadmium & $1.36 \mathrm{E}-05$ & $\mathrm{~kg} / \mathrm{Mg}$ \\
Chromium & $1.50 \mathrm{E}-05$ & $\mathrm{~kg} / \mathrm{Mg}$ \\
Nickel & $2.58 \mathrm{E}-05$ & $\mathrm{~kg} / \mathrm{Mg}$ \\
Lead & $1.31 \mathrm{E}-04$ & $\mathrm{~kg} / \mathrm{Mg}$ \\
CDD/CDF & $3.31 \mathrm{E}-08$ & $\mathrm{~kg} / \mathrm{Mg}$ \\
Mercury & $2.80 \mathrm{E}-04$ & $\mathrm{~kg} / \mathrm{Mg}$ \\
NOx & $2.75 \mathrm{E}-01$ & $\mathrm{~kg} / \mathrm{Mg}$ \\
Sulphur dioxide & $2.77 \mathrm{E}-01$ & $\mathrm{~kg} / \mathrm{Mg}$ \\
Hydrogen & & \\
chloride & $1.06 \mathrm{E}-01$ & $\mathrm{~kg} / \mathrm{Mg}$ \\
Particulate matter & $3.11 \mathrm{E}-02$ & $\mathrm{~kg} / \mathrm{Mg}$ \\
CO & $2.31 \mathrm{E}-02$ & $\mathrm{~kg} / \mathrm{Mg}$ \\
\hline
\end{tabular}


Table S9. Names, operating parameters and modeling units used for the important components in the Aspen Plus simulations.

\begin{tabular}{|c|c|c|c|c|}
\hline Sr. No. & Equipment & Temperature (K) & Pressure (atm) & Modeled as \\
\hline 1 & Gasification reactor $^{21}$ & 1573 & 26 & RGibbs reactor \\
\hline 2 & Fast pyrolysis reactor 22,24 & 773 & 1 & RStoic reactor \\
\hline 5 & Preheater & 372 & 1 & HeatX \\
\hline 6 & Air compressor & - & 26 & Compressor \\
\hline 8 & Hydrotreater $^{22,} 25$ & 672 & 172 & RYield reactor \\
\hline 9 & Hydrocracker $^{22}$ & 700 & 87 & RYield reactor \\
\hline 10 & Gasoline cooler & 283 & 32 & HeatX \\
\hline 11 & Diesel cooler & 283 & 32 & HeatX \\
\hline 13 & Steam methane reformer ${ }^{26}$ & 1123 & 45 & RStoic reactor \\
\hline 14 & Water-gas shift reactor ${ }^{25}$ & 573 & 29 & RStoic reactor \\
\hline 15 & Distillation Column & - & 23 & RadFrac \\
\hline 16 & Condenser & 344 & 1.7 & - \\
\hline 17 & Reboiler & 590 & 1.7 & - \\
\hline 18 & Combustor & 823 & 1 & RGibbs reactor \\
\hline
\end{tabular}




\section{S2.2 LCA and TEA parameters and results}

Table S10. Factors used for calculation of the total project investment and capital investment values. $^{27}$

\begin{tabular}{lll}
\hline Type & \% & Factor \\
\hline total purchased equipment cost (TPEC) & $100 \%$ & 1 \\
purchased equipment installation & $39 \%$ of TPEC & 0.39 \\
instrumentation and controls & $26 \%$ of TPEC & 0.26 \\
piping & $31 \%$ of TPEC & 0.31 \\
electrical systems & $10 \%$ of TPEC & 0.1 \\
buildings (including services) & $29 \%$ of TPEC & 0.29 \\
yard improvements & $12 \%$ of TPEC & 0.12 \\
service facilities & $55 \%$ of TPEC & 0.55 \\
total installed cost (TIC) & & 3.02 \\
indirect costs & & \\
engineering & $32 \%$ of TPEC & 0.32 \\
construction & $34 \%$ of TPEC & 0.34 \\
legal and contractors fees & $23 \%$ of TPEC & 0.23 \\
project contingency & $34 \%$ of TPEC & 0.34 \\
total indirect costs & & $\mathbf{1 . 2 3}$ \\
land & $5 \%$ of TPEC & 0.06 \\
working capital (WC) & 0.05 \\
total project investment (TPI) & & \\
\hline
\end{tabular}


Table S11. Annualized cost distribution

\begin{tabular}{lrcrr}
\hline PP Processing System & $\begin{array}{l}\text { Annualized } \\
\text { CAPEX }\end{array}$ & $\begin{array}{c}\text { Annual } \\
\text { Annual OPEX } \\
(\$ \mathrm{MM})\end{array}$ & \multicolumn{2}{l}{$\begin{array}{l}\text { Annual } \\
\text { Taxes }\end{array}$} \\
\hline Fast pyrolysis & 51.02 & 30.61 & 8.16 & 10.20 \\
Gasification & 49.09 & 32.89 & 8.20 & 9.82 \\
Incineration (with CC) & 27.13 & 58.84 & 8.60 & 5.43 \\
Incineration & 0.00 & 80.75 & 11.80 & 7.45 \\
Landfilling (with CC) & 36.15 & 48.19 & 8.43 & 7.23 \\
Landfilling & 0.00 & 75.47 & 13.21 & 11.32 \\
\hline
\end{tabular}

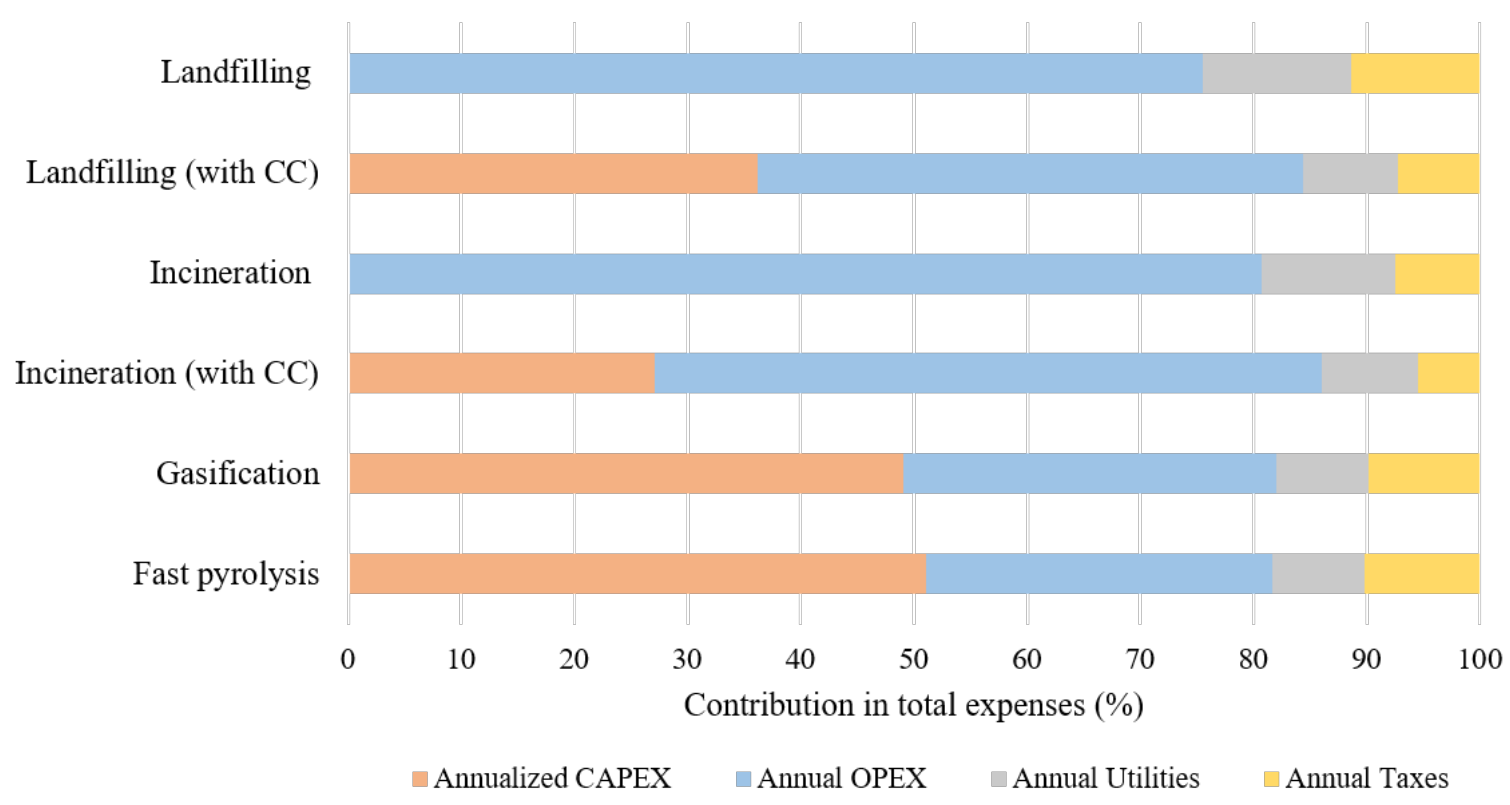

Figure S1. The annual production costs for the systems analyzed. Both incineration and landfilling are represented by cases with and without the capital costs (CC). 
Table S12. NPV calculation for fast pyrolysis.

\begin{tabular}{ccccc}
\hline year & $\begin{array}{c}\text { cash flow } \\
(\$)\end{array}$ & $\begin{array}{c}\text { present value } \\
(\$)\end{array}$ & $\begin{array}{c}\text { cumulated NPV } \\
(\$)\end{array}$ & $\begin{array}{c}\text { cumulated NPV } \\
(\$ M M)\end{array}$ \\
\hline 0 & 101567210.2 & $-1.02 \mathrm{E}+08$ & -101567210.2 & -101.567 \\
1 & 0 & 0 & -101567210.2 & -101.567 \\
2 & 0 & 0 & -101567210.2 & -101.567 \\
3 & 10090000 & 7580766.3 & -93986443.84 & -93.9864 \\
4 & 40360000 & 27566423 & -66420020.78 & -66.42 \\
5 & 40360000 & 25060385 & -41359636.18 & -41.3596 \\
6 & 40360000 & 22782168 & -18577468.36 & -18.5775 \\
7 & 40360000 & 20711062 & 2133593.287 & 2.133593 \\
8 & 40360000 & 18828238 & 20961831.15 & 20.96183 \\
9 & 40360000 & 17116580 & 38078411.03 & 38.07841 \\
10 & 40360000 & 15560527 & 53638938.19 & 53.63894 \\
11 & 40360000 & 14145934 & 67784871.97 & 67.78487 \\
12 & 40360000 & 12859940 & 80644811.78 & 80.64481 \\
13 & 40360000 & 11690854 & 92335666.14 & 92.33567 \\
14 & 40360000 & 10628049 & 102963715.6 & 102.9637 \\
15 & 40360000 & 9661863.1 & 112625578.7 & 112.6256 \\
16 & 40360000 & 8783511.9 & 121409090.6 & 121.4091 \\
17 & 40360000 & 7985010.8 & 129394101.4 & 129.3941 \\
18 & 40360000 & 7259100.8 & 136653202.2 & 136.6532 \\
19 & 40360000 & 6599182.5 & 143252384.7 & 143.2524 \\
20 & 40360000 & 5999256.8 & 149251641.5 & 149.2516 \\
\hline & & & &
\end{tabular}




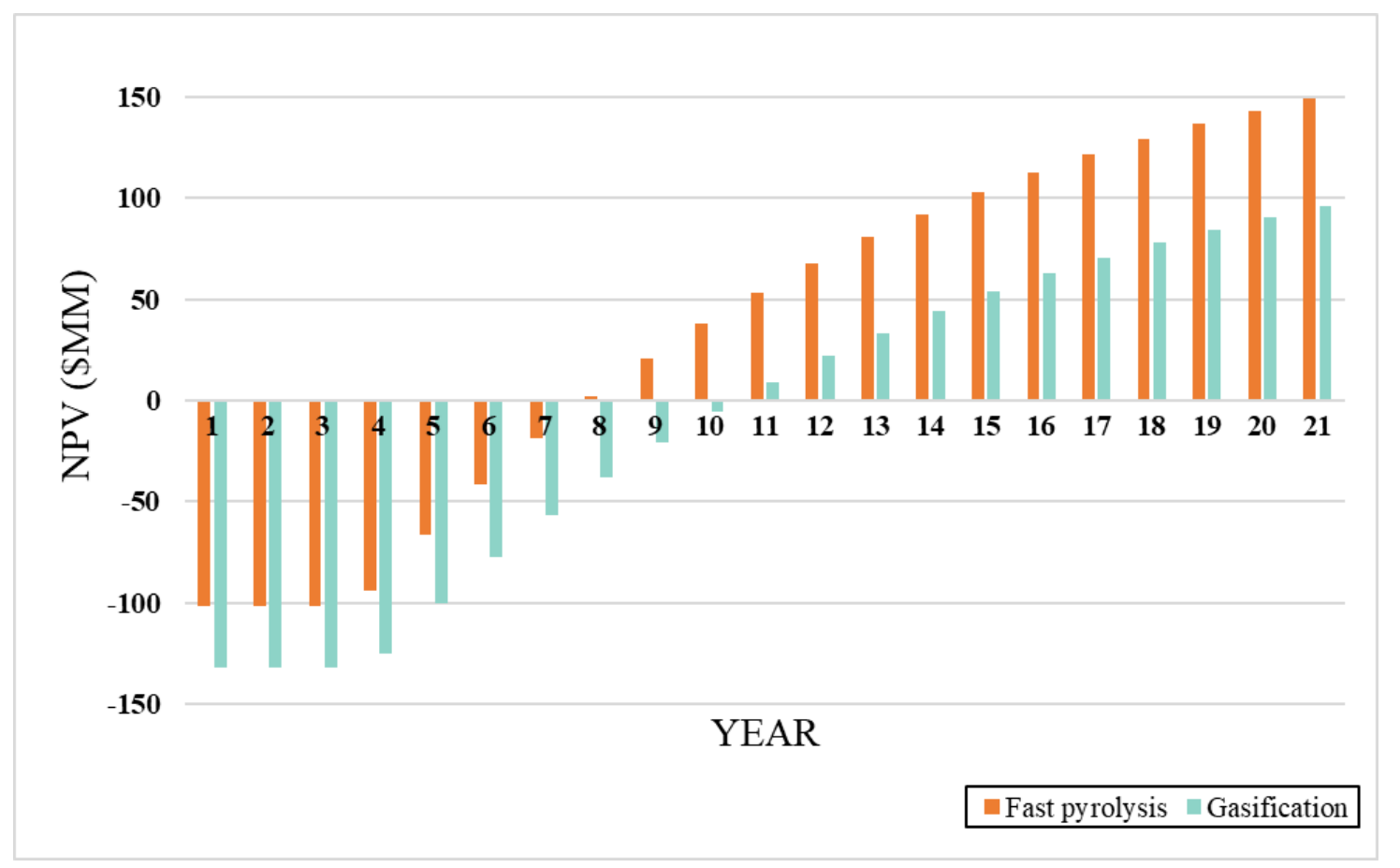

Figure S2. Comparison of the NPV for the gasification and fast pyrolysis pathways.

Table S13. Sensitivity analysis parameters for fast pyrolysis.

\begin{tabular}{llccccc}
\hline Parameter & Units & $\begin{array}{c}\text { Base } \\
\text { case }\end{array}$ & $\begin{array}{c}\text { Lower } \\
\text { extreme }\end{array}$ & $\begin{array}{c}\text { Corresponding } \\
\text { NPV }(\$ M M)\end{array}$ & $\begin{array}{c}\text { Higher } \\
\text { extreme }\end{array}$ & $\begin{array}{c}\text { Corresponding } \\
\text { NPV (\$MM) }\end{array}$ \\
\hline Electricity cost & $\$ / \mathrm{kWh}$ & 0.05 & 0.052 & 144.5 & 0.07 & 162.8 \\
Plant capacity & ton/hr & 12 & 6 & 93.1 & 25 & 183.8 \\
Waste PP price & $\$ /$ ton & 200 & - & - & 1000 & 310.53 \\
Plant life & years & 20 & 10 & 53.6 & 30 & 186.5 \\
Discount rate & $\%$ & 10 & 5 & 300.2 & 15 & 65.5 \\
\hline
\end{tabular}

Table S14. Sensitivity analysis parameters for gasification.

\begin{tabular}{llccccc}
\hline Parameter & Units & $\begin{array}{c}\text { Base } \\
\text { case }\end{array}$ & $\begin{array}{c}\text { Lower } \\
\text { extreme }\end{array}$ & $\begin{array}{c}\text { Corresponding } \\
\text { NPV }(\$ M M)\end{array}$ & $\begin{array}{c}\text { Higher } \\
\text { extreme }\end{array}$ & $\begin{array}{c}\text { Corresponding } \\
\text { NPV (\$MM) }\end{array}$ \\
\hline Electricity cost & \$/kWh & 0.05 & 0.052 & 92.2 & 0.07 & 124.8 \\
Plant capacity & ton/hr & 12 & 6 & 22.9 & 25 & 140.9 \\
Discount rate & $\%$ & 10 & 5 & 129.5 & 15 & 9.1 \\
Plant life & years & 20 & 10 & 53.6 & 30 & 186.5 \\
Waste PP price & \$/ton & 200 & - & - & 1000 & 257.53 \\
\hline
\end{tabular}




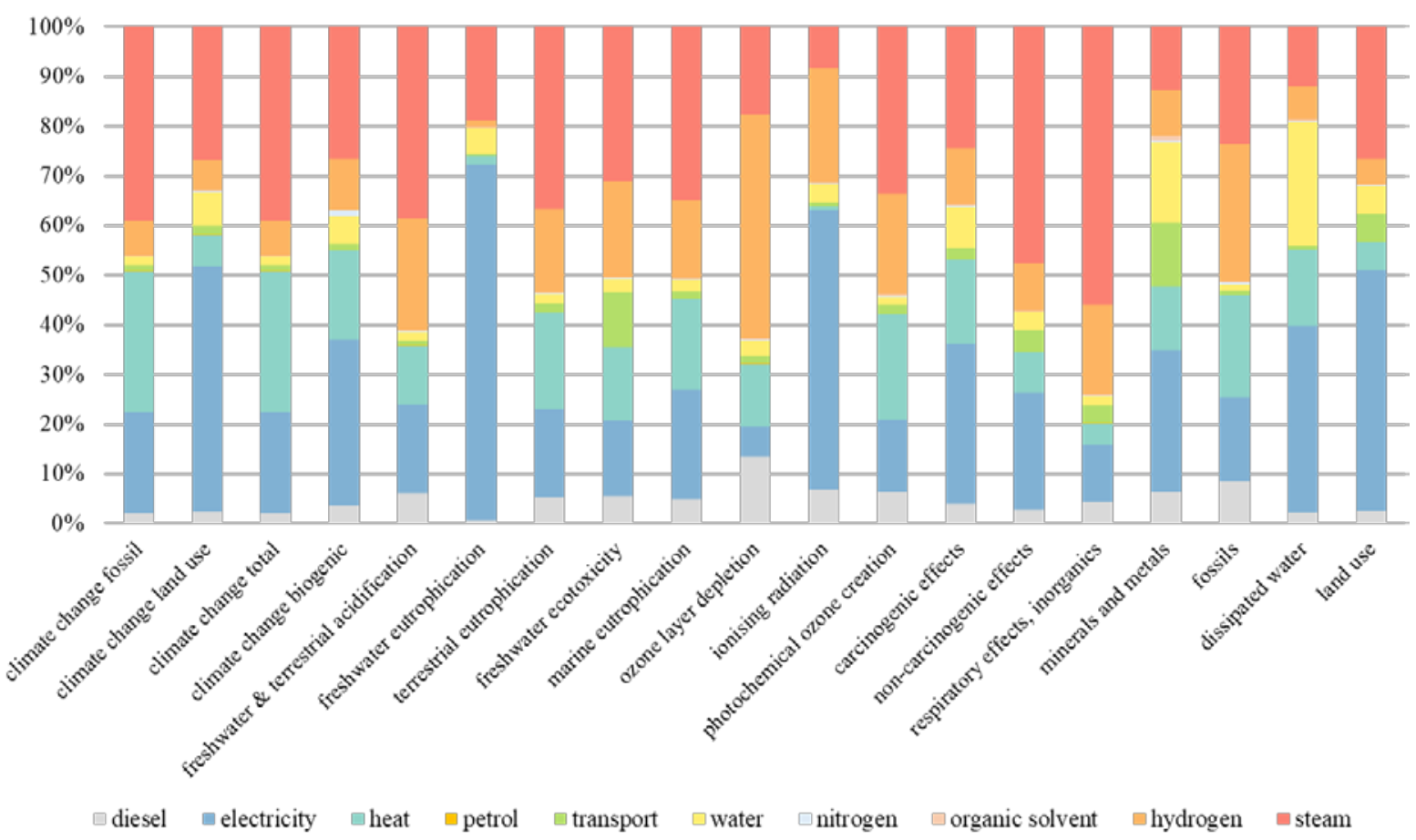

Figure S3. ILCD contribution analysis for fast pyrolysis.

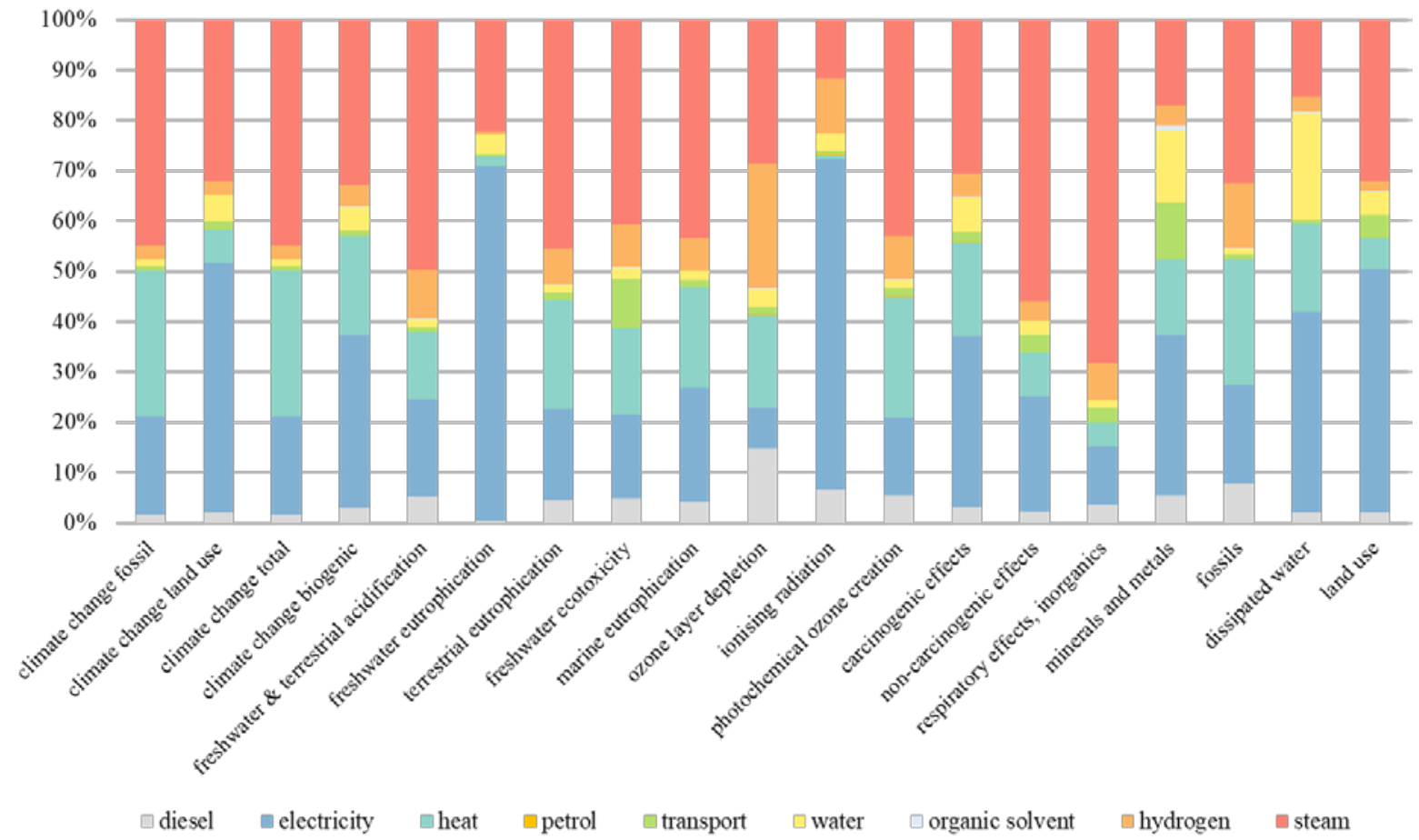

Figure S4. ILCD contribution analysis for gasification. 


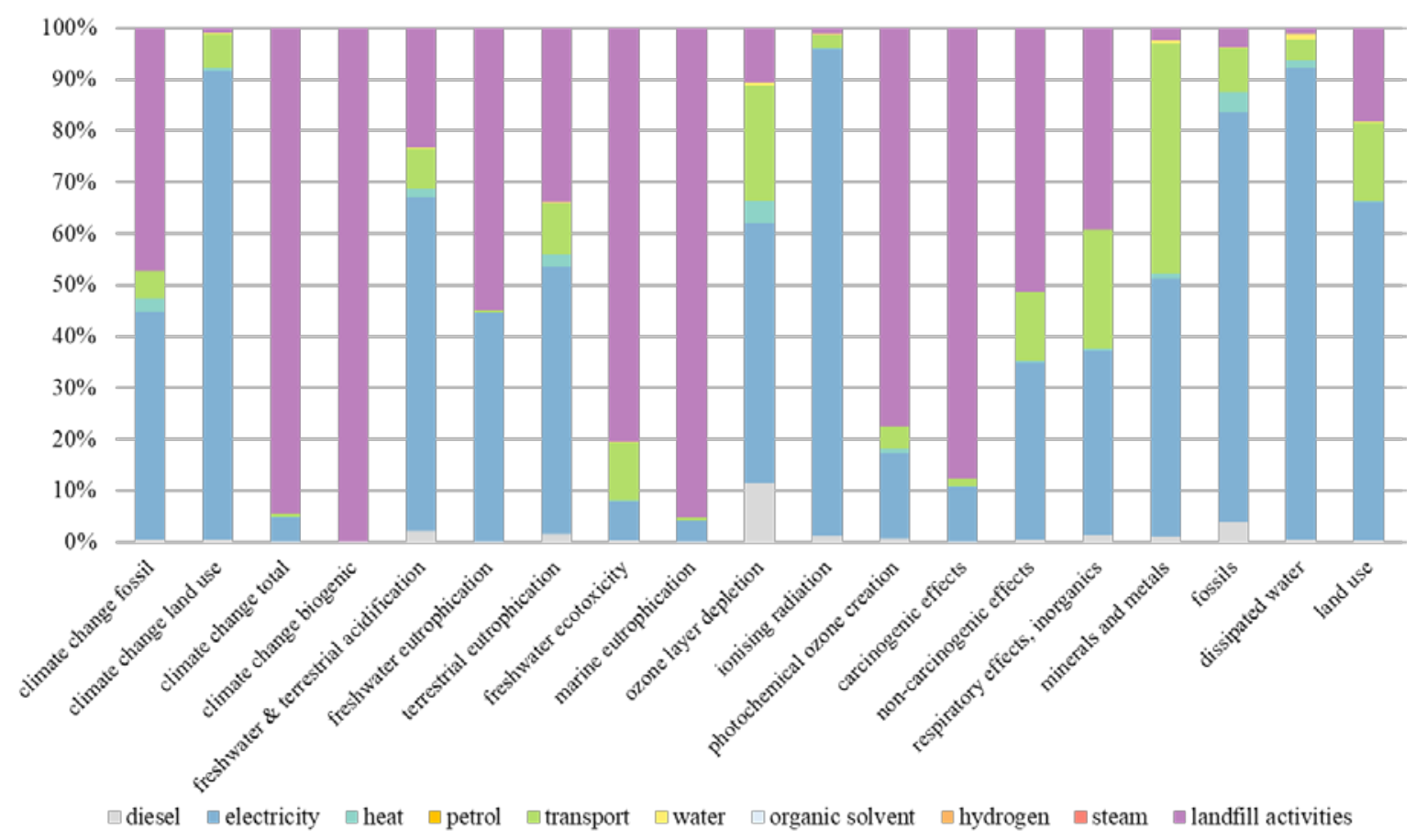

Figure S5. ILCD contribution analysis for landfilling.

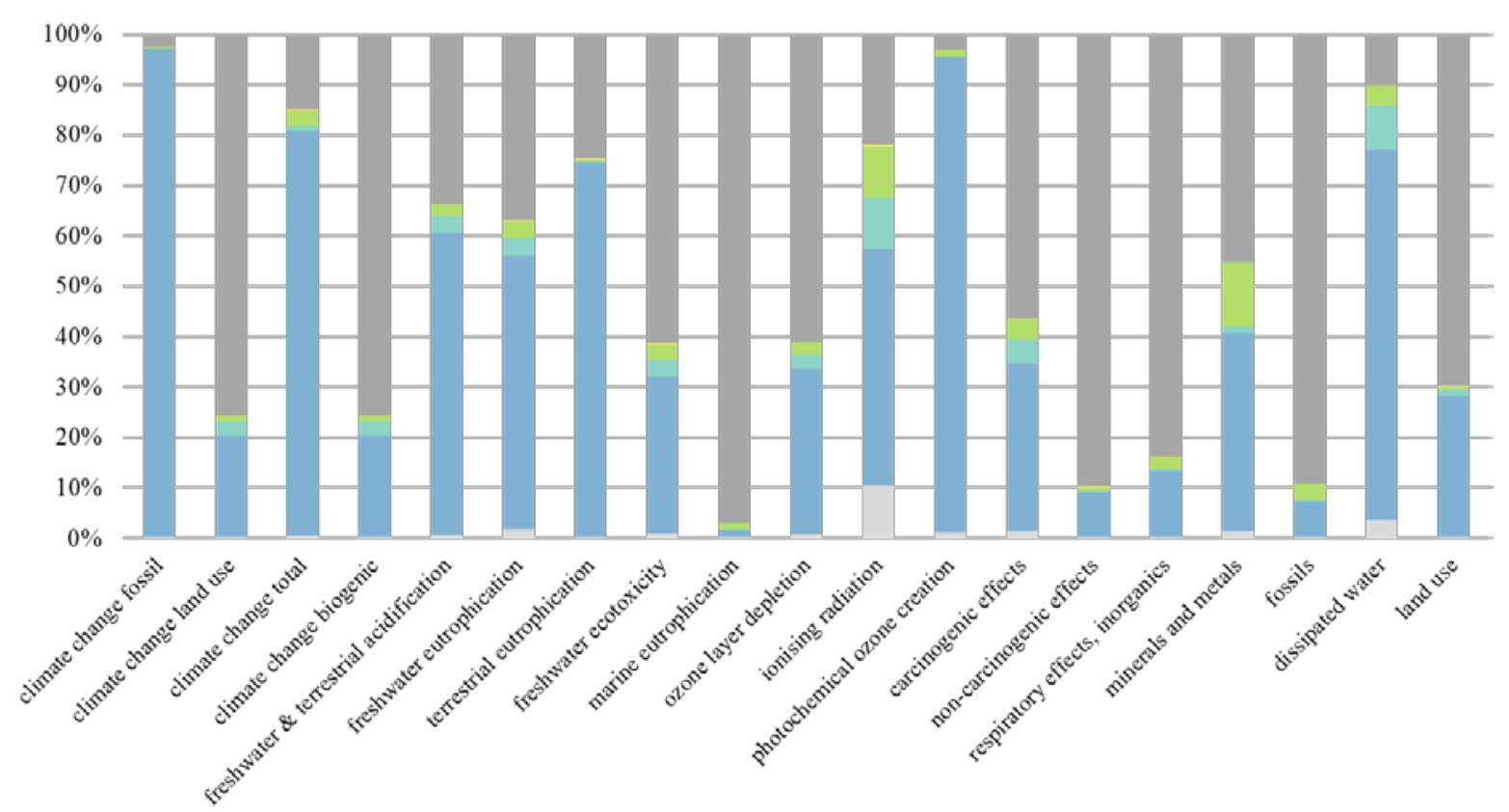

$\square$ diesel $\square$ electricity $\square$ heat $\square$ petrol $\square$ transport $\square$ water $\square$ organic solvent $\square$ hydrogen $\square$ steam $\square$ incinerator activities

Figure S6. ILCD contribution analysis for incineration. 
Table S15. ReCiPe results for fast pyrolysis of 1 ton of waste PP.

\begin{tabular}{|c|c|c|c|c|c|c|c|c|c|c|}
\hline Impact category & diesel & electricity & heat & petrol & transport & water & helium & $\begin{array}{l}\text { organic } \\
\text { solvent }\end{array}$ & hydrogen & steam \\
\hline Agricultural land occupation ( $\left.\mathrm{m}^{2} \mathrm{a}\right)$ & $1.85 \mathrm{E}+00$ & $8.51 \mathrm{E}+01$ & $4.53 \mathrm{E}+00$ & $1.67 \mathrm{E}-02$ & $9.36 \mathrm{E}-01$ & $7.63 \mathrm{E}+00$ & $2.29 \mathrm{E}-01$ & $2.90 \mathrm{E}-01$ & $4.75 \mathrm{E}+00$ & $2.11 \mathrm{E}+01$ \\
\hline GHG emissions ( $\mathrm{kg} \mathrm{CO}_{2}$-Eq) & $1.45 \mathrm{E}+02$ & $1.49 \mathrm{E}+03$ & $2.04 \mathrm{E}+03$ & $1.24 \mathrm{E}+00$ & $9.31 \mathrm{E}+01$ & $1.25 \mathrm{E}+02$ & $7.31 \mathrm{E}+00$ & $4.42 \mathrm{E}+00$ & $5.18 \mathrm{E}+02$ & $2.85 \mathrm{E}+03$ \\
\hline Fossil depletion (kg oil-Eq) & $3.37 \mathrm{E}+02$ & $4.56 \mathrm{E}+02$ & $1.04 \mathrm{E}+03$ & $2.49 \mathrm{E}+00$ & $3.60 \mathrm{E}+01$ & $3.67 \mathrm{E}+01$ & $1.33 \mathrm{E}+01$ & $7.27 \mathrm{E}+00$ & $1.12 \mathrm{E}+03$ & $9.74 \mathrm{E}+02$ \\
\hline \multicolumn{11}{|l|}{ Freshwater ecotoxicity (kg 1,4-DCB- } \\
\hline Eq) & $1.46 \mathrm{E}+00$ & $2.69 \mathrm{E}+01$ & $1.01 \mathrm{E}+01$ & $1.26 \mathrm{E}-02$ & 7.48E-01 & $4.61 \mathrm{E}+00$ & $1.85 \mathrm{E}-01$ & $1.94 \mathrm{E}-01$ & $3.47 \mathrm{E}+00$ & $1.56 \mathrm{E}+01$ \\
\hline Freshwater eutrophication (kg P-Eq) & $1.14 \mathrm{E}-02$ & $1.32 \mathrm{E}+00$ & 3.30E-02 & $9.54 \mathrm{E}-05$ & 7.84E-03 & $9.41 \mathrm{E}-02$ & $1.87 \mathrm{E}-03$ & $1.00 \mathrm{E}-03$ & 2.89E-02 & 3.46E-01 \\
\hline Human toxicity (kg 1,4-DCB-Eq) & $2.54 \mathrm{E}+01$ & $8.44 \mathrm{E}+02$ & $7.90 \mathrm{E}+01$ & $2.31 \mathrm{E}-01$ & $3.11 \mathrm{E}+01$ & $7.06 \mathrm{E}+01$ & $2.12 \mathrm{E}+00$ & $1.58 \mathrm{E}+00$ & $7.96 \mathrm{E}+01$ & $3.87 \mathrm{E}+02$ \\
\hline Ionizing radiation (kg U235-Eq) & $6.22 \mathrm{E}+01$ & $5.03 \mathrm{E}+02$ & $6.65 \mathrm{E}+00$ & 4.57E-01 & $7.14 \mathrm{E}+00$ & $3.31 \mathrm{E}+01$ & 5.38E-01 & $1.32 \mathrm{E}+00$ & $2.06 \mathrm{E}+02$ & $7.47 \mathrm{E}+01$ \\
\hline Marine ecotoxicity (kg 1,4-DCB-Eq) & $1.29 \mathrm{E}+00$ & $2.31 \mathrm{E}+01$ & $6.54 \mathrm{E}+00$ & $1.16 \mathrm{E}-02$ & $1.04 \mathrm{E}+00$ & $4.05 \mathrm{E}+00$ & 1.19E-01 & $1.71 \mathrm{E}-01$ & $3.22 \mathrm{E}+00$ & $1.33 \mathrm{E}+01$ \\
\hline Marine eutrophication (kg N-Eq) & 2.63E-02 & 3.81E-01 & 9.73E-02 & $1.96 \mathrm{E}-04$ & $9.35 \mathrm{E}-03$ & 3.50E-02 & $1.18 \mathrm{E}-03$ & 8.55E-04 & 8.00E-02 & 2.32E-01 \\
\hline Metal depletion (kg Fe-Eq) & $3.21 \mathrm{E}+00$ & $1.20 \mathrm{E}+01$ & $1.14 \mathrm{E}+01$ & $2.54 \mathrm{E}-02$ & $3.12 \mathrm{E}+00$ & $6.77 \mathrm{E}+00$ & $1.87 \mathrm{E}-01$ & 3.95E-01 & $5.86 \mathrm{E}+00$ & $8.19 \mathrm{E}+00$ \\
\hline Natural land transformation $\left(\mathrm{m}^{2}\right)$ & $-5.69 \mathrm{E}-03$ & $-5.19 \mathrm{E}-02$ & $-2.15 \mathrm{E}-02$ & $-2.54 \mathrm{E}-05$ & $-1.27 \mathrm{E}-02$ & $-8.07 \mathrm{E}-03$ & $-2.44 \mathrm{E}-04$ & $-2.65 \mathrm{E}-04$ & $-7.08 \mathrm{E}-03$ & $-5.65 \mathrm{E}-02$ \\
\hline Ozone depletion (kg CFC-11-Eq) & $1.78 \mathrm{E}-04$ & 1.13E-04 & 1.79E-04 & $1.31 \mathrm{E}-06$ & $1.76 \mathrm{E}-05$ & 5.33E-05 & $2.42 \mathrm{E}-06$ & 5.18E-06 & $5.94 \mathrm{E}-04$ & 2.35E-04 \\
\hline \multicolumn{11}{|l|}{ Particulate matter formation (kg PM10- } \\
\hline Eq) & $4.10 \mathrm{E}-01$ & $4.68 \mathrm{E}+00$ & 8.55E-01 & 3.37E-03 & $1.64 \mathrm{E}-01$ & 3.75E-01 & $1.36 \mathrm{E}-02$ & $1.08 \mathrm{E}-02$ & $1.42 \mathrm{E}+00$ & $2.98 \mathrm{E}+00$ \\
\hline \multicolumn{11}{|l|}{ Photochemical oxidant formation (kg } \\
\hline NMVOC) & $9.62 \mathrm{E}-01$ & $2.21 \mathrm{E}+00$ & $3.19 \mathrm{E}+00$ & $7.28 \mathrm{E}-03$ & $2.88 \mathrm{E}-01$ & $2.51 \mathrm{E}-01$ & 2.97E-02 & $6.76 \mathrm{E}-02$ & $3.09 \mathrm{E}+00$ & $5.11 \mathrm{E}+00$ \\
\hline Terrestrial acidification (kg SO${ }_{2}$-Eq) & $1.35 \mathrm{E}+00$ & $3.88 \mathrm{E}+00$ & $2.56 \mathrm{E}+00$ & 1.15E-02 & $2.36 \mathrm{E}-01$ & 3.88E-01 & $3.00 \mathrm{E}-02$ & $3.16 \mathrm{E}-02$ & $4.88 \mathrm{E}+00$ & $8.40 \mathrm{E}+00$ \\
\hline \multicolumn{11}{|l|}{ Terrestrial ecotoxicity (kg 1,4-DCB- } \\
\hline Eq) & 2.79E-02 & 8.47E-02 & 7.49E-02 & $2.50 \mathrm{E}-04$ & 5.89E-02 & 1.59E-02 & $1.53 \mathrm{E}-03$ & $7.01 \mathrm{E}-04$ & 9.89E-02 & 3.76E-01 \\
\hline Urban land occupation ( $\left.\mathrm{m}^{2} \mathrm{a}\right)$ & $1.03 \mathrm{E}+01$ & $1.93 \mathrm{E}+02$ & $2.29 \mathrm{E}+01$ & 1.03E-01 & $1.71 \mathrm{E}+01$ & $2.41 \mathrm{E}+01$ & $6.81 \mathrm{E}-01$ & 7.36E-01 & $1.88 \mathrm{E}+01$ & $1.20 \mathrm{E}+02$ \\
\hline Water depletion $\left(\mathrm{m}^{3}\right)$ & 4.37E-01 & $7.24 \mathrm{E}+00$ & $3.00 \mathrm{E}+00$ & $3.20 \mathrm{E}-03$ & $1.57 \mathrm{E}-01$ & $4.81 \mathrm{E}+00$ & $2.46 \mathrm{E}-02$ & $6.81 \mathrm{E}-02$ & $1.29 \mathrm{E}+00$ & $2.32 \mathrm{E}+00$ \\
\hline
\end{tabular}


Table S16. ReCiPe results for gasification of 1 ton of waste PP.

\begin{tabular}{|c|c|c|c|c|c|c|c|c|c|}
\hline Impact category & diesel & electricity & heat & petrol & transport & water & $\begin{array}{l}\text { organic } \\
\text { solvent }\end{array}$ & hydrogen & steam \\
\hline Agricultural land occupation $\left(\mathrm{m}^{2} \mathrm{a}\right)$ & $1.85 \mathrm{E}+00$ & $1.06 \mathrm{E}+02$ & $6.04 \mathrm{E}+00$ & $1.67 \mathrm{E}-02$ & $9.36 \mathrm{E}-01$ & $7.63 \mathrm{E}+00$ & $2.90 \mathrm{E}-01$ & $2.37 \mathrm{E}+00$ & $3.17 \mathrm{E}+01$ \\
\hline GHG emissions ( $\mathrm{kg} \mathrm{CO}_{2}$-Eq) & $1.45 \mathrm{E}+02$ & $1.86 \mathrm{E}+03$ & $2.72 E+03$ & $1.24 \mathrm{E}+00$ & $9.31 \mathrm{E}+01$ & $1.25 \mathrm{E}+02$ & $4.42 \mathrm{E}+00$ & $2.59 \mathrm{E}+02$ & $4.28 \mathrm{E}+03$ \\
\hline Fossil depletion (kg oil-Eq) & $3.37 \mathrm{E}+02$ & $5.71 \mathrm{E}+02$ & $1.39 \mathrm{E}+03$ & $2.49 \mathrm{E}+00$ & $3.60 \mathrm{E}+01$ & $3.67 \mathrm{E}+01$ & $7.27 \mathrm{E}+00$ & $5.60 \mathrm{E}+02$ & $1.46 \mathrm{E}+03$ \\
\hline \multicolumn{10}{|l|}{ Freshwater ecotoxicity (kg 1,4-DCB- } \\
\hline Eq) & $1.46 \mathrm{E}+00$ & $3.36 \mathrm{E}+01$ & $1.34 \mathrm{E}+01$ & $1.26 \mathrm{E}-02$ & 7.48E-01 & $4.61 \mathrm{E}+00$ & $1.94 \mathrm{E}-01$ & $1.73 \mathrm{E}+00$ & $2.33 \mathrm{E}+01$ \\
\hline Human toxicity (kg 1,4-DCB-Eq) & $2.54 \mathrm{E}+01$ & $1.05 \mathrm{E}+03$ & $1.05 \mathrm{E}+02$ & 2.31E-01 & $3.11 \mathrm{E}+01$ & $7.06 \mathrm{E}+01$ & $1.58 \mathrm{E}+00$ & $3.98 \mathrm{E}+01$ & $5.81 \mathrm{E}+02$ \\
\hline Ionizing radiation (kg U235-Eq) & $6.22 \mathrm{E}+01$ & $6.29 \mathrm{E}+02$ & $8.87 \mathrm{E}+00$ & 4.57E-01 & $7.14 \mathrm{E}+00$ & $3.31 \mathrm{E}+01$ & $1.32 \mathrm{E}+00$ & $1.03 \mathrm{E}+02$ & $1.12 \mathrm{E}+02$ \\
\hline Marine ecotoxicity (kg 1,4-DCB-Eq) & $1.29 \mathrm{E}+00$ & $2.89 \mathrm{E}+01$ & $8.72 \mathrm{E}+00$ & $1.16 \mathrm{E}-02$ & $1.04 \mathrm{E}+00$ & $4.05 \mathrm{E}+00$ & $1.71 \mathrm{E}-01$ & $1.61 \mathrm{E}+00$ & $2.00 \mathrm{E}+01$ \\
\hline Marine eutrophication (kg N-Eq) & 2.63E-02 & 4.77E-01 & $1.30 \mathrm{E}-01$ & $1.96 \mathrm{E}-04$ & $9.35 \mathrm{E}-03$ & 3.50E-02 & 8.55E-04 & $4.00 \mathrm{E}-02$ & $3.48 \mathrm{E}-01$ \\
\hline Metal depletion (kg Fe-Eq) & $3.21 \mathrm{E}+00$ & $1.50 \mathrm{E}+01$ & $1.51 \mathrm{E}+01$ & $2.54 \mathrm{E}-02$ & $3.12 \mathrm{E}+00$ & $6.77 \mathrm{E}+00$ & 3.95E-01 & $2.93 \mathrm{E}+00$ & $1.23 \mathrm{E}+01$ \\
\hline Natural land transformation $\left(\mathrm{m}^{2}\right)$ & $-5.69 \mathrm{E}-03$ & $-6.49 \mathrm{E}-02$ & $-2.87 \mathrm{E}-02$ & $-2.54 \mathrm{E}-05$ & $-1.27 \mathrm{E}-02$ & $-8.07 \mathrm{E}-03$ & $-2.65 \mathrm{E}-04$ & $-3.54 \mathrm{E}-03$ & $-8.48 \mathrm{E}-02$ \\
\hline Ozone depletion (kg CFC-11-Eq) & $1.78 \mathrm{E}-04$ & $1.41 \mathrm{E}-04$ & 2.38E-04 & $1.31 \mathrm{E}-06$ & $1.76 \mathrm{E}-05$ & 5.33E-05 & $5.18 \mathrm{E}-06$ & 2.97E-04 & 3.52E-04 \\
\hline Eq) & 4.10E-01 & $5.85 \mathrm{E}+00$ & $1.14 \mathrm{E}+00$ & 3.37E-03 & $1.64 \mathrm{E}-01$ & 3.75E-01 & $1.08 \mathrm{E}-02$ & 7.10E-01 & $4.46 \mathrm{E}+00$ \\
\hline \multicolumn{10}{|l|}{ Photochemical oxidant formation (kg } \\
\hline NMVOC) & $9.62 \mathrm{E}-01$ & $2.77 \mathrm{E}+00$ & $4.25 \mathrm{E}+00$ & $7.28 \mathrm{E}-03$ & $2.88 \mathrm{E}-01$ & $2.51 \mathrm{E}-01$ & $6.76 \mathrm{E}-02$ & $1.55 \mathrm{E}+00$ & $7.67 \mathrm{E}+00$ \\
\hline Terrestrial acidification (kg SO${ }_{2}$-Eq) & $1.35 \mathrm{E}+00$ & $4.85 \mathrm{E}+00$ & $3.41 \mathrm{E}+00$ & 1.15E-02 & 2.36E-01 & 3.88E-01 & 3.16E-02 & $2.44 \mathrm{E}+00$ & $1.26 \mathrm{E}+01$ \\
\hline \multicolumn{10}{|l|}{ Terrestrial ecotoxicity (kg 1,4-DCB- } \\
\hline Eq) & 2.79E-02 & $1.06 \mathrm{E}-01$ & 9.99E-02 & $2.50 \mathrm{E}-04$ & 5.89E-02 & 1.59E-02 & 7.01E-04 & 4.95E-02 & 5.63E-01 \\
\hline Urban land occupation ( $\left.\mathrm{m}^{2} \mathrm{a}\right)$ & $1.03 \mathrm{E}+01$ & $2.41 \mathrm{E}+02$ & $3.05 \mathrm{E}+01$ & 1.03E-01 & $1.71 \mathrm{E}+01$ & $2.41 \mathrm{E}+01$ & 7.36E-01 & $9.39 \mathrm{E}+00$ & $1.80 \mathrm{E}+02$ \\
\hline Water depletion $\left(\mathrm{m}^{3}\right)$ & 4.37E-01 & $9.06 \mathrm{E}+00$ & $4.00 \mathrm{E}+00$ & $3.20 \mathrm{E}-03$ & $1.57 \mathrm{E}-01$ & $4.81 \mathrm{E}+00$ & $6.81 \mathrm{E}-02$ & 6.47E-01 & $3.49 \mathrm{E}+00$ \\
\hline
\end{tabular}


Table S17. ReCiPe results for landfilling of 1 ton of waste PP.

\begin{tabular}{|c|c|c|c|c|c|c|c|c|c|c|}
\hline Impact category & diesel & electricity & heat & petrol & transport & water & $\begin{array}{l}\text { organic } \\
\text { solvent }\end{array}$ & hydrogen & steam & $\begin{array}{c}\text { landfill } \\
\text { activities }\end{array}$ \\
\hline Agricultural land occupation ( $\left.\mathrm{m}^{2} \mathrm{a}\right)$ & $9.24 \mathrm{E}-02$ & $4.26 \mathrm{E}+01$ & $9.06 \mathrm{E}-02$ & $0.00 \mathrm{E}+00$ & $9.36 \mathrm{E}-01$ & 7.63E-02 & $0.00 \mathrm{E}+00$ & $0.00 \mathrm{E}+00$ & $0.00 \mathrm{E}+00$ & $1.04 \mathrm{E}+01$ \\
\hline GHG emissions ( $\mathrm{kg} \mathrm{CO}_{2}$-Eq) & $7.27 \mathrm{E}+00$ & $7.46 \mathrm{E}+02$ & $4.08 \mathrm{E}+01$ & $0.00 \mathrm{E}+00$ & $9.31 \mathrm{E}+01$ & $1.25 \mathrm{E}+00$ & $0.00 \mathrm{E}+00$ & $0.00 \mathrm{E}+00$ & $0.00 \mathrm{E}+00$ & $1.01 \mathrm{E}+04$ \\
\hline Fossil depletion (kg oil-Eq) & $1.69 \mathrm{E}+01$ & $2.28 \mathrm{E}+02$ & $2.09 \mathrm{E}+01$ & $0.00 \mathrm{E}+00$ & $3.60 \mathrm{E}+01$ & 3.67E-01 & $0.00 \mathrm{E}+00$ & $0.00 \mathrm{E}+00$ & $0.00 \mathrm{E}+00$ & $1.63 \mathrm{E}+01$ \\
\hline \multicolumn{11}{|l|}{ Freshwater ecotoxicity (kg 1,4-DCB- } \\
\hline Eq) & 7.29E-02 & $1.34 \mathrm{E}+01$ & 2.02E-01 & $0.00 \mathrm{E}+00$ & 7.48E-01 & 4.61E-02 & $0.00 \mathrm{E}+00$ & $0.00 \mathrm{E}+00$ & $0.00 \mathrm{E}+00$ & $1.84 \mathrm{E}+03$ \\
\hline Freshwater eutrophication (kg P-Eq) & $5.69 \mathrm{E}-04$ & 6.59E-01 & 6.59E-04 & $0.00 \mathrm{E}+00$ & 7.84E-03 & $9.41 \mathrm{E}-04$ & $0.00 \mathrm{E}+00$ & $0.00 \mathrm{E}+00$ & $0.00 \mathrm{E}+00$ & 8.11E-01 \\
\hline Human toxicity (kg 1,4-DCB-Eq) & $1.27 \mathrm{E}+00$ & $4.22 \mathrm{E}+02$ & $1.58 \mathrm{E}+00$ & $0.00 \mathrm{E}+00$ & $3.11 \mathrm{E}+01$ & 7.06E-01 & $0.00 \mathrm{E}+00$ & $0.00 \mathrm{E}+00$ & $0.00 \mathrm{E}+00$ & $5.07 \mathrm{E}+03$ \\
\hline Ionizing radiation (kg U235-Eq) & $3.11 \mathrm{E}+00$ & $2.52 \mathrm{E}+02$ & 1.33E-01 & $0.00 \mathrm{E}+00$ & $7.14 \mathrm{E}+00$ & 3.31E-01 & $0.00 \mathrm{E}+00$ & $0.00 \mathrm{E}+00$ & $0.00 \mathrm{E}+00$ & $3.06 \mathrm{E}+00$ \\
\hline Marine ecotoxicity (kg 1,4-DCB-Eq) & $6.47 \mathrm{E}-02$ & $1.16 \mathrm{E}+01$ & $1.31 \mathrm{E}-01$ & $0.00 \mathrm{E}+00$ & $1.04 \mathrm{E}+00$ & 4.05E-02 & $0.00 \mathrm{E}+00$ & $0.00 \mathrm{E}+00$ & $0.00 \mathrm{E}+00$ & $1.58 \mathrm{E}+03$ \\
\hline Marine eutrophication (kg N-Eq) & $1.31 \mathrm{E}-03$ & 1.91E-01 & $1.95 \mathrm{E}-03$ & $0.00 \mathrm{E}+00$ & $9.35 \mathrm{E}-03$ & 3.50E-04 & $0.00 \mathrm{E}+00$ & $0.00 \mathrm{E}+00$ & $0.00 \mathrm{E}+00$ & $3.33 \mathrm{E}+01$ \\
\hline Metal depletion (kg Fe-Eq) & $1.60 \mathrm{E}-01$ & $6.01 \mathrm{E}+00$ & 2.27E-01 & $0.00 \mathrm{E}+00$ & $3.12 \mathrm{E}+00$ & $6.77 \mathrm{E}-02$ & $0.00 \mathrm{E}+00$ & $0.00 \mathrm{E}+00$ & $0.00 \mathrm{E}+00$ & 7.66E-01 \\
\hline Natural land transformation $\left(\mathrm{m}^{2}\right)$ & $-2.84 \mathrm{E}-04$ & $-2.60 \mathrm{E}-02$ & $-4.30 \mathrm{E}-04$ & $0.00 \mathrm{E}+00$ & $-1.27 \mathrm{E}-02$ & $-8.07 \mathrm{E}-05$ & $0.00 \mathrm{E}+00$ & $0.00 \mathrm{E}+00$ & $0.00 \mathrm{E}+00$ & $-4.23 \mathrm{E}-04$ \\
\hline Ozone depletion (kg CFC-11-Eq) & 8.92E-06 & 5.66E-05 & 3.57E-06 & $0.00 \mathrm{E}+00$ & $1.76 \mathrm{E}-05$ & 5.33E-07 & $0.00 \mathrm{E}+00$ & $0.00 \mathrm{E}+00$ & $0.00 \mathrm{E}+00$ & 8.34E-06 \\
\hline \multicolumn{11}{|l|}{ Particulate matter formation (kg PM10- } \\
\hline Eq) & 2.05E-02 & $2.34 \mathrm{E}+00$ & $1.71 \mathrm{E}-02$ & $0.00 \mathrm{E}+00$ & $1.64 \mathrm{E}-01$ & 3.75E-03 & $0.00 \mathrm{E}+00$ & $0.00 \mathrm{E}+00$ & $0.00 \mathrm{E}+00$ & 2.63E-01 \\
\hline \multicolumn{11}{|l|}{ Photochemical oxidant formation (kg } \\
\hline NMVOC) & $4.81 \mathrm{E}-02$ & $1.11 \mathrm{E}+00$ & $6.38 \mathrm{E}-02$ & $0.00 \mathrm{E}+00$ & $2.88 \mathrm{E}-01$ & $2.51 \mathrm{E}-03$ & $0.00 \mathrm{E}+00$ & $0.00 \mathrm{E}+00$ & $0.00 \mathrm{E}+00$ & $5.23 \mathrm{E}+00$ \\
\hline Terrestrial acidification (kg SO${ }_{2}$-Eq) & $6.75 \mathrm{E}-02$ & $1.94 \mathrm{E}+00$ & 5.11E-02 & $0.00 \mathrm{E}+00$ & $2.36 \mathrm{E}-01$ & 3.88E-03 & $0.00 \mathrm{E}+00$ & $0.00 \mathrm{E}+00$ & $0.00 \mathrm{E}+00$ & $6.94 \mathrm{E}-01$ \\
\hline \multicolumn{11}{|l|}{ Terrestrial ecotoxicity (kg 1,4-DCB- } \\
\hline Eq) & $1.40 \mathrm{E}-03$ & 4.23E-02 & $1.50 \mathrm{E}-03$ & $0.00 \mathrm{E}+00$ & 5.89E-02 & 1.59E-04 & $0.00 \mathrm{E}+00$ & $0.00 \mathrm{E}+00$ & $0.00 \mathrm{E}+00$ & $1.01 \mathrm{E}-01$ \\
\hline Urban land occupation ( $\left.\mathrm{m}^{2} \mathrm{a}\right)$ & 5.15E-01 & $9.63 \mathrm{E}+01$ & 4.58E-01 & $0.00 \mathrm{E}+00$ & $1.71 \mathrm{E}+01$ & $2.41 \mathrm{E}-01$ & $0.00 \mathrm{E}+00$ & $0.00 \mathrm{E}+00$ & $0.00 \mathrm{E}+00$ & $1.67 \mathrm{E}+01$ \\
\hline Water depletion $\left(\mathrm{m}^{3}\right)$ & 2.19E-02 & $3.62 \mathrm{E}+00$ & $6.00 \mathrm{E}-02$ & $0.00 \mathrm{E}+00$ & $1.57 \mathrm{E}-01$ & 4.81E-02 & $0.00 \mathrm{E}+00$ & $0.00 \mathrm{E}+00$ & $0.00 \mathrm{E}+00$ & 4.14E-02 \\
\hline
\end{tabular}


Table S18. ReCiPe results for incineration of 1 ton of waste PP.

\begin{tabular}{|c|c|c|c|c|c|c|c|c|c|c|}
\hline Impact category & diesel & electricity & heat & petrol & transport & water & $\begin{array}{l}\text { organic } \\
\text { solvent }\end{array}$ & hydrogen & steam & $\begin{array}{c}\text { incineration } \\
\text { activities }\end{array}$ \\
\hline Agricultural land occupation $\left(\mathrm{m}^{2} \mathrm{a}\right)$ & 1.85E-01 & $8.51 \mathrm{E}+01$ & 4.53E-01 & $0.00 \mathrm{E}+00$ & $9.36 \mathrm{E}-01$ & 7.63E-02 & $0.00 \mathrm{E}+00$ & $0.00 \mathrm{E}+00$ & $0.00 \mathrm{E}+00$ & $1.08 \mathrm{E}+01$ \\
\hline GHG emissions ( $\mathrm{kg} \mathrm{CO}_{2}$-Eq) & $1.45 \mathrm{E}+01$ & $1.49 \mathrm{E}+03$ & $2.04 \mathrm{E}+02$ & $0.00 \mathrm{E}+00$ & $9.31 \mathrm{E}+01$ & $1.25 \mathrm{E}+00$ & $0.00 \mathrm{E}+00$ & $0.00 \mathrm{E}+00$ & $0.00 \mathrm{E}+00$ & $5.73 \mathrm{E}+03$ \\
\hline Fossil depletion (kg oil-Eq) & $3.37 \mathrm{E}+01$ & $4.56 \mathrm{E}+02$ & $1.04 \mathrm{E}+02$ & $0.00 \mathrm{E}+00$ & $3.60 \mathrm{E}+01$ & 3.67E-01 & $0.00 \mathrm{E}+00$ & $0.00 \mathrm{E}+00$ & $0.00 \mathrm{E}+00$ & $9.70 \mathrm{E}+01$ \\
\hline \multicolumn{11}{|l|}{ Freshwater ecotoxicity (kg 1,4-DCB- } \\
\hline $\mathrm{Eq)}$ & $1.46 \mathrm{E}-01$ & $2.69 \mathrm{E}+01$ & $1.01 \mathrm{E}+00$ & $0.00 \mathrm{E}+00$ & 7.48E-01 & 4.61E-02 & $0.00 \mathrm{E}+00$ & $0.00 \mathrm{E}+00$ & $0.00 \mathrm{E}+00$ & $3.28 \mathrm{E}+03$ \\
\hline Freshwater eutrophication (kg P-Eq) & $1.14 \mathrm{E}-03$ & $1.32 \mathrm{E}+00$ & 3.30E-03 & $0.00 \mathrm{E}+00$ & 7.84E-03 & $9.41 \mathrm{E}-04$ & $0.00 \mathrm{E}+00$ & $0.00 \mathrm{E}+00$ & $0.00 \mathrm{E}+00$ & 4.37E-01 \\
\hline Human toxicity (kg 1,4-DCB-Eq) & $2.54 \mathrm{E}+00$ & $8.44 \mathrm{E}+02$ & $7.90 \mathrm{E}+00$ & $0.00 \mathrm{E}+00$ & $3.11 \mathrm{E}+01$ & 7.06E-01 & $0.00 \mathrm{E}+00$ & $0.00 \mathrm{E}+00$ & $0.00 \mathrm{E}+00$ & $4.31 \mathrm{E}+03$ \\
\hline Ionizing radiation (kg U235-Eq) & $6.22 \mathrm{E}+00$ & $5.03 \mathrm{E}+02$ & $6.65 \mathrm{E}-01$ & $0.00 \mathrm{E}+00$ & $7.14 \mathrm{E}+00$ & 3.31E-01 & $0.00 \mathrm{E}+00$ & $0.00 \mathrm{E}+00$ & $0.00 \mathrm{E}+00$ & $1.63 \mathrm{E}+01$ \\
\hline Marine ecotoxicity (kg 1,4-DCB-Eq) & $1.29 \mathrm{E}-01$ & $2.31 \mathrm{E}+01$ & $6.54 \mathrm{E}-01$ & $0.00 \mathrm{E}+00$ & $1.04 \mathrm{E}+00$ & $4.05 \mathrm{E}-02$ & $0.00 \mathrm{E}+00$ & $0.00 \mathrm{E}+00$ & $0.00 \mathrm{E}+00$ & $3.17 \mathrm{E}+03$ \\
\hline Marine eutrophication (kg N-Eq) & 2.63E-03 & 3.81E-01 & $9.73 \mathrm{E}-03$ & $0.00 \mathrm{E}+00$ & $9.35 \mathrm{E}-03$ & 3.50E-04 & $0.00 \mathrm{E}+00$ & $0.00 \mathrm{E}+00$ & $0.00 \mathrm{E}+00$ & 5.63E-01 \\
\hline Metal depletion (kg Fe-Eq) & 3.21E-01 & $1.20 \mathrm{E}+01$ & $1.14 \mathrm{E}+00$ & $0.00 \mathrm{E}+00$ & $3.12 \mathrm{E}+00$ & $6.77 \mathrm{E}-02$ & $0.00 \mathrm{E}+00$ & $0.00 \mathrm{E}+00$ & $0.00 \mathrm{E}+00$ & $7.35 \mathrm{E}+01$ \\
\hline Natural land transformation $\left(\mathrm{m}^{2}\right)$ & $-5.69 \mathrm{E}-04$ & $-5.19 \mathrm{E}-02$ & $-2.15 \mathrm{E}-03$ & $0.00 \mathrm{E}+00$ & $-1.27 \mathrm{E}-02$ & $-8.07 \mathrm{E}-05$ & $0.00 \mathrm{E}+00$ & $0.00 \mathrm{E}+00$ & $0.00 \mathrm{E}+00$ & $-2.80 \mathrm{E}-02$ \\
\hline Ozone depletion (kg CFC-11-Eq) & $1.78 \mathrm{E}-05$ & 1.13E-04 & 1.79E-05 & $0.00 \mathrm{E}+00$ & $1.76 \mathrm{E}-05$ & 5.33E-07 & $0.00 \mathrm{E}+00$ & $0.00 \mathrm{E}+00$ & $0.00 \mathrm{E}+00$ & 4.35E-05 \\
\hline \multicolumn{11}{|l|}{ Particulate matter formation (kg PM10- } \\
\hline Eq) & $4.10 \mathrm{E}-02$ & $4.68 \mathrm{E}+00$ & 8.55E-02 & $0.00 \mathrm{E}+00$ & $1.64 \mathrm{E}-01$ & 3.75E-03 & $0.00 \mathrm{E}+00$ & $0.00 \mathrm{E}+00$ & $0.00 \mathrm{E}+00$ & $1.14 \mathrm{E}+00$ \\
\hline \multicolumn{11}{|l|}{ Photochemical oxidant formation (kg } \\
\hline NMVOC) & $9.62 \mathrm{E}-02$ & $2.21 \mathrm{E}+00$ & 3.19E-01 & $0.00 \mathrm{E}+00$ & $2.88 \mathrm{E}-01$ & $2.51 \mathrm{E}-03$ & $0.00 \mathrm{E}+00$ & $0.00 \mathrm{E}+00$ & $0.00 \mathrm{E}+00$ & $3.78 \mathrm{E}+00$ \\
\hline Terrestrial acidification (kg SO${ }_{2}$-Eq) & 1.35E-01 & $3.88 \mathrm{E}+00$ & $2.56 \mathrm{E}-01$ & $0.00 \mathrm{E}+00$ & $2.36 \mathrm{E}-01$ & $3.88 \mathrm{E}-03$ & $0.00 \mathrm{E}+00$ & $0.00 \mathrm{E}+00$ & $0.00 \mathrm{E}+00$ & $2.64 \mathrm{E}+00$ \\
\hline \multicolumn{11}{|l|}{ Terrestrial ecotoxicity (kg 1,4-DCB- } \\
\hline Eq) & 2.79E-03 & 8.47E-02 & 7.49E-03 & $0.00 \mathrm{E}+00$ & 5.89E-02 & 1.59E-04 & $0.00 \mathrm{E}+00$ & $0.00 \mathrm{E}+00$ & $0.00 \mathrm{E}+00$ & $9.58 \mathrm{E}-01$ \\
\hline Urban land occupation $\left(\mathrm{m}^{2} \mathrm{a}\right)$ & $1.03 \mathrm{E}+00$ & $1.93 \mathrm{E}+02$ & $2.29 \mathrm{E}+00$ & $0.00 \mathrm{E}+00$ & $1.71 \mathrm{E}+01$ & $2.41 \mathrm{E}-01$ & $0.00 \mathrm{E}+00$ & $0.00 \mathrm{E}+00$ & $0.00 \mathrm{E}+00$ & $5.18 \mathrm{E}+01$ \\
\hline Water depletion $\left(\mathrm{m}^{3}\right)$ & 4.37E-02 & $7.24 \mathrm{E}+00$ & $3.00 \mathrm{E}-01$ & $0.00 \mathrm{E}+00$ & 1.57E-01 & $4.81 \mathrm{E}-02$ & $0.00 \mathrm{E}+00$ & $0.00 \mathrm{E}+00$ & $0.00 \mathrm{E}+00$ & $1.80 \mathrm{E}+01$ \\
\hline
\end{tabular}


Table S19. ILCD, 2.0 results for fast pyrolysis of 1 ton of waste PP.

\begin{tabular}{|c|c|c|c|c|c|c|c|c|c|c|}
\hline Impact category & diesel & electricity & heat & petrol & transport & water & helium & $\begin{array}{l}\text { organic } \\
\text { solvent }\end{array}$ & hydrogen & steam \\
\hline climate change fossil & $1.50 \mathrm{E}+02$ & $1.52 \mathrm{E}+03$ & $2.13 \mathrm{E}+03$ & $1.27 \mathrm{E}+00$ & $9.41 \mathrm{E}+01$ & $1.28 \mathrm{E}+02$ & $8.14 \mathrm{E}+00$ & $4.58 \mathrm{E}+00$ & $5.33 \mathrm{E}+02$ & $2.92 \mathrm{E}+03$ \\
\hline climate change land use & 4.22E-02 & 8.70E-01 & $1.10 \mathrm{E}-01$ & $2.70 \mathrm{E}-04$ & $3.22 \mathrm{E}-02$ & 1.15E-01 & $6.82 \mathrm{E}-03$ & $2.25 \mathrm{E}-03$ & $1.10 \mathrm{E}-01$ & 4.70E-01 \\
\hline climate change total & $1.50 \mathrm{E}+02$ & $1.53 \mathrm{E}+03$ & $2.13 \mathrm{E}+03$ & $1.27 \mathrm{E}+00$ & $9.41 \mathrm{E}+01$ & $1.28 \mathrm{E}+02$ & $8.17 \mathrm{E}+00$ & $4.58 \mathrm{E}+00$ & $5.33 \mathrm{E}+02$ & $2.92 \mathrm{E}+03$ \\
\hline climate change biogenic & 8.93E-02 & 8.12E-01 & 4.35E-01 & $5.51 \mathrm{E}-04$ & 3.22E-02 & $1.40 \mathrm{E}-01$ & $2.46 \mathrm{E}-02$ & 4.30E-03 & $2.49 \mathrm{E}-01$ & $6.48 \mathrm{E}-01$ \\
\hline freshwater \& terrestrial acidification & $1.77 \mathrm{E}+00$ & $5.08 \mathrm{E}+00$ & $3.36 \mathrm{E}+00$ & $1.51 \mathrm{E}-02$ & 3.09E-01 & 5.09E-01 & 3.94E-02 & 4.15E-02 & $6.41 \mathrm{E}+00$ & $1.10 \mathrm{E}+01$ \\
\hline freshwater eutrophication & $1.10 \mathrm{E}-02$ & $1.32 \mathrm{E}+00$ & 3.27E-02 & $9.24 \mathrm{E}-05$ & $7.80 \mathrm{E}-03$ & $9.40 \mathrm{E}-02$ & $1.87 \mathrm{E}-03$ & $9.92 \mathrm{E}-04$ & $2.76 \mathrm{E}-02$ & 3.45E-01 \\
\hline terrestrial eutrophication & $2.37 \mathrm{E}+00$ & $7.72 \mathrm{E}+00$ & $8.58 \mathrm{E}+00$ & $1.78 \mathrm{E}-02$ & 7.52E-01 & $8.80 \mathrm{E}-01$ & $6.54 \mathrm{E}-02$ & $6.58 \mathrm{E}-02$ & $7.39 \mathrm{E}+00$ & $1.61 \mathrm{E}+01$ \\
\hline freshwater ecotoxicity & $1.61 \mathrm{E}+02$ & $4.39 \mathrm{E}+02$ & $4.27 \mathrm{E}+02$ & $1.38 \mathrm{E}+00$ & $3.23 \mathrm{E}+02$ & $7.47 \mathrm{E}+01$ & $8.30 \mathrm{E}+00$ & $4.26 \mathrm{E}+00$ & $5.62 \mathrm{E}+02$ & $8.98 \mathrm{E}+02$ \\
\hline marine eutrophication & 2.17E-01 & $9.60 \mathrm{E}-01$ & 7.94E-01 & $1.63 \mathrm{E}-03$ & $6.92 \mathrm{E}-02$ & $9.89 \mathrm{E}-02$ & $6.38 \mathrm{E}-03$ & $6.04 \mathrm{E}-03$ & $6.80 \mathrm{E}-01$ & $1.53 \mathrm{E}+00$ \\
\hline ozone layer depletion & $2.26 \mathrm{E}-04$ & $1.00 \mathrm{E}-04$ & $2.12 \mathrm{E}-04$ & $1.66 \mathrm{E}-06$ & $2.22 \mathrm{E}-05$ & $5.21 \mathrm{E}-05$ & $2.91 \mathrm{E}-06$ & 5.53E-06 & $7.51 \mathrm{E}-04$ & 2.94E-04 \\
\hline ionizing radiation & $6.22 \mathrm{E}+01$ & $5.02 \mathrm{E}+02$ & $6.64 \mathrm{E}+00$ & 4.57E-01 & $7.14 \mathrm{E}+00$ & $3.30 \mathrm{E}+01$ & 5.37E-01 & $1.32 \mathrm{E}+00$ & $2.06 \mathrm{E}+02$ & $7.46 \mathrm{E}+01$ \\
\hline photochemical ozone creation & $9.62 \mathrm{E}-01$ & $2.23 \mathrm{E}+00$ & $3.22 \mathrm{E}+00$ & $7.28 \mathrm{E}-03$ & 2.88E-01 & $2.52 \mathrm{E}-01$ & 2.95E-02 & $4.11 \mathrm{E}-02$ & $3.09 \mathrm{E}+00$ & $5.11 \mathrm{E}+00$ \\
\hline carcinogenic effects & 1.15E-06 & $9.65 \mathrm{E}-06$ & 4.99E-06 & 7.99E-09 & $6.98 \mathrm{E}-07$ & $2.44 \mathrm{E}-06$ & $1.03 \mathrm{E}-07$ & $6.50 \mathrm{E}-08$ & 3.34E-06 & 7.26E-06 \\
\hline non-carcinogenic effects & $1.14 \mathrm{E}-05$ & $9.24 \mathrm{E}-05$ & 3.25E-05 & $9.17 \mathrm{E}-08$ & $1.79 \mathrm{E}-05$ & $1.40 \mathrm{E}-05$ & $6.81 \mathrm{E}-07$ & $5.91 \mathrm{E}-07$ & 3.76E-05 & $1.88 \mathrm{E}-04$ \\
\hline respiratory effects, inorganics & $9.83 \mathrm{E}-06$ & 2.53E-05 & $9.58 \mathrm{E}-06$ & $9.51 \mathrm{E}-08$ & $8.10 \mathrm{E}-06$ & 3.93E-06 & $2.21 \mathrm{E}-07$ & 2.49E-07 & 4.03E-05 & $1.24 \mathrm{E}-04$ \\
\hline minerals and metals & $7.46 \mathrm{E}-04$ & $3.41 \mathrm{E}-03$ & $1.52 \mathrm{E}-03$ & 3.19E-06 & $1.52 \mathrm{E}-03$ & $1.93 \mathrm{E}-03$ & 3.74E-05 & $1.16 \mathrm{E}-04$ & $1.09 \mathrm{E}-03$ & $1.53 \mathrm{E}-03$ \\
\hline fossils & $1.37 \mathrm{E}+04$ & $2.80 \mathrm{E}+04$ & $3.35 \mathrm{E}+04$ & $1.01 \mathrm{E}+02$ & $1.51 \mathrm{E}+03$ & $2.15 \mathrm{E}+03$ & $4.46 \mathrm{E}+02$ & $2.97 \mathrm{E}+02$ & $4.54 \mathrm{E}+04$ & $3.87 \mathrm{E}+04$ \\
\hline dissipated water & $1.88 \mathrm{E}+01$ & $3.11 \mathrm{E}+02$ & $1.29 \mathrm{E}+02$ & 1.37E-01 & $6.76 \mathrm{E}+00$ & $2.07 \mathrm{E}+02$ & $1.06 \mathrm{E}+00$ & $2.92 \mathrm{E}+00$ & $5.56 \mathrm{E}+01$ & $9.98 \mathrm{E}+01$ \\
\hline land use & $1.62 E+03$ & $3.06 \mathrm{E}+04$ & $3.56 \mathrm{E}+03$ & $1.50 \mathrm{E}+01$ & $3.55 \mathrm{E}+03$ & $3.65 E+03$ & $1.03 E+02$ & $1.20 \mathrm{E}+02$ & $3.15 E+03$ & $1.69 \mathrm{E}+04$ \\
\hline
\end{tabular}


Table S20. ILCD, 2.0 results for gasification of 1 ton of waste PP.

\begin{tabular}{|c|c|c|c|c|c|c|c|c|c|}
\hline Impact category & diesel & electricity & heat & petrol & transport & water & $\begin{array}{l}\text { organic } \\
\text { solvent }\end{array}$ & hydrogen & steam \\
\hline climate change fossil & $1.50 \mathrm{E}+02$ & $1.90 \mathrm{E}+03$ & $2.83 \mathrm{E}+03$ & $1.27 \mathrm{E}+00$ & $9.41 \mathrm{E}+01$ & $1.28 \mathrm{E}+02$ & $4.58 \mathrm{E}+00$ & $2.66 \mathrm{E}+02$ & $4.38 \mathrm{E}+03$ \\
\hline climate change land use & 4.22E-02 & $1.09 \mathrm{E}+00$ & $1.47 \mathrm{E}-01$ & $2.70 \mathrm{E}-04$ & 3.22E-02 & 1.15E-01 & 2.25E-03 & 5.51E-02 & 7.04E-01 \\
\hline climate change total & $1.50 \mathrm{E}+02$ & $1.91 \mathrm{E}+03$ & $2.83 \mathrm{E}+03$ & $1.27 \mathrm{E}+00$ & $9.41 \mathrm{E}+01$ & $1.28 \mathrm{E}+02$ & $4.58 \mathrm{E}+00$ & $2.67 \mathrm{E}+02$ & $4.38 \mathrm{E}+03$ \\
\hline climate change biogenic & 8.93E-02 & $1.02 \mathrm{E}+00$ & $5.80 \mathrm{E}-01$ & $5.51 \mathrm{E}-04$ & 3.22E-02 & $1.40 \mathrm{E}-01$ & 4.30E-03 & $1.24 \mathrm{E}-01$ & $9.72 \mathrm{E}-01$ \\
\hline freshwater \& terrestrial acidification & $1.77 \mathrm{E}+00$ & $6.35 \mathrm{E}+00$ & $4.48 \mathrm{E}+00$ & $1.51 \mathrm{E}-02$ & 3.09E-01 & 5.09E-01 & 4.15E-02 & $3.20 \mathrm{E}+00$ & $1.65 \mathrm{E}+01$ \\
\hline freshwater eutrophication & $1.10 \mathrm{E}-02$ & $1.65 \mathrm{E}+00$ & 4.36E-02 & $9.24 \mathrm{E}-05$ & 7.80E-03 & $9.40 \mathrm{E}-02$ & $9.92 \mathrm{E}-04$ & 1.38E-02 & 5.17E-01 \\
\hline terrestrial eutrophication & $2.37 \mathrm{E}+00$ & $9.65 \mathrm{E}+00$ & $1.14 \mathrm{E}+01$ & $1.78 \mathrm{E}-02$ & 7.52E-01 & 8.80E-01 & $6.58 \mathrm{E}-02$ & $3.70 \mathrm{E}+00$ & $2.42 \mathrm{E}+01$ \\
\hline freshwater ecotoxicity & $1.61 \mathrm{E}+02$ & $5.48 \mathrm{E}+02$ & $5.69 \mathrm{E}+02$ & $1.38 \mathrm{E}+00$ & $3.23 \mathrm{E}+02$ & $7.47 \mathrm{E}+01$ & $4.26 \mathrm{E}+00$ & $2.81 \mathrm{E}+02$ & $1.35 \mathrm{E}+03$ \\
\hline marine eutrophication & 2.17E-01 & $1.20 \mathrm{E}+00$ & $1.06 \mathrm{E}+00$ & 1.63E-03 & 6.92E-02 & $9.89 \mathrm{E}-02$ & $6.04 \mathrm{E}-03$ & $3.40 \mathrm{E}-01$ & $2.29 \mathrm{E}+00$ \\
\hline ozone layer depletion & $2.26 \mathrm{E}-04$ & $1.25 \mathrm{E}-04$ & 2.83E-04 & 1.66E-06 & $2.22 \mathrm{E}-05$ & $5.21 \mathrm{E}-05$ & 5.53E-06 & $3.76 \mathrm{E}-04$ & $4.41 \mathrm{E}-04$ \\
\hline ionizing radiation & $6.22 \mathrm{E}+01$ & $6.28 \mathrm{E}+02$ & $8.86 \mathrm{E}+00$ & 4.57E-01 & $7.14 \mathrm{E}+00$ & $3.30 \mathrm{E}+01$ & $1.32 \mathrm{E}+00$ & $1.03 \mathrm{E}+02$ & $1.12 \mathrm{E}+02$ \\
\hline photochemical ozone creation & $9.62 \mathrm{E}-01$ & $2.78 \mathrm{E}+00$ & $4.29 \mathrm{E}+00$ & 7.28E-03 & $2.88 \mathrm{E}-01$ & 2.52E-01 & 4.11E-02 & $1.55 \mathrm{E}+00$ & $7.66 \mathrm{E}+00$ \\
\hline carcinogenic effects & $1.15 \mathrm{E}-06$ & $1.21 \mathrm{E}-05$ & 6.66E-06 & 7.99E-09 & $6.98 \mathrm{E}-07$ & 2.44E-06 & $6.50 \mathrm{E}-08$ & 1.67E-06 & 1.09E-05 \\
\hline non-carcinogenic effects & $1.14 \mathrm{E}-05$ & $1.16 \mathrm{E}-04$ & 4.34E-05 & $9.17 \mathrm{E}-08$ & 1.79E-05 & $1.40 \mathrm{E}-05$ & $5.91 \mathrm{E}-07$ & $1.88 \mathrm{E}-05$ & 2.82E-04 \\
\hline respiratory effects, inorganics & $9.83 \mathrm{E}-06$ & 3.16E-05 & $1.28 \mathrm{E}-05$ & $9.51 \mathrm{E}-08$ & 8.10E-06 & 3.93E-06 & 2.49E-07 & 2.01E-05 & $1.86 \mathrm{E}-04$ \\
\hline minerals and metals & 7.46E-04 & 4.27E-03 & 2.03E-03 & 3.19E-06 & $1.52 \mathrm{E}-03$ & $1.93 \mathrm{E}-03$ & $1.16 \mathrm{E}-04$ & 5.43E-04 & 2.29E-03 \\
\hline fossils & $1.37 \mathrm{E}+04$ & $3.50 \mathrm{E}+04$ & $4.46 \mathrm{E}+04$ & $1.01 \mathrm{E}+02$ & $1.51 \mathrm{E}+03$ & $2.15 \mathrm{E}+03$ & $2.97 \mathrm{E}+02$ & $2.27 \mathrm{E}+04$ & $5.81 \mathrm{E}+04$ \\
\hline dissipated water & $1.88 \mathrm{E}+01$ & $3.89 \mathrm{E}+02$ & $1.72 \mathrm{E}+02$ & 1.37E-01 & $6.76 \mathrm{E}+00$ & $2.07 \mathrm{E}+02$ & $2.92 \mathrm{E}+00$ & $2.78 \mathrm{E}+01$ & $1.50 \mathrm{E}+02$ \\
\hline land use & $1.62 E+03$ & $3.82 E+04$ & $4.74 \mathrm{E}+03$ & $1.50 \mathrm{E}+01$ & $3.55 E+03$ & $3.65 \mathrm{E}+03$ & $1.20 \mathrm{E}+02$ & $1.58 \mathrm{E}+03$ & $2.53 E+04$ \\
\hline
\end{tabular}


Table S21. ILCD, 2.0 results for landfilling of 1 ton of waste PP.

\begin{tabular}{|c|c|c|c|c|c|c|c|c|c|c|}
\hline Impact category & diesel & electricity & heat & petrol & transport & water & $\begin{array}{l}\text { organic } \\
\text { solvent }\end{array}$ & hydrogen & steam & landfill activities \\
\hline climate change fossil & $7.51 \mathrm{E}+00$ & $7.62 \mathrm{E}+02$ & $4.25 \mathrm{E}+01$ & $0.00 \mathrm{E}+00$ & $9.41 \mathrm{E}+01$ & $1.28 \mathrm{E}+00$ & $0.00 \mathrm{E}+00$ & $0.00 \mathrm{E}+00$ & $0.00 \mathrm{E}+00$ & $8.10 \mathrm{E}+02$ \\
\hline climate change land use & $2.11 \mathrm{E}-03$ & 4.35E-01 & $2.20 \mathrm{E}-03$ & $0.00 \mathrm{E}+00$ & $3.22 \mathrm{E}-02$ & $1.15 \mathrm{E}-03$ & $0.00 \mathrm{E}+00$ & $0.00 \mathrm{E}+00$ & $0.00 \mathrm{E}+00$ & 4.35E-03 \\
\hline climate change total & $7.52 \mathrm{E}+00$ & $7.63 \mathrm{E}+02$ & $4.25 \mathrm{E}+01$ & $0.00 \mathrm{E}+00$ & $9.41 \mathrm{E}+01$ & $1.28 \mathrm{E}+00$ & $0.00 \mathrm{E}+00$ & $0.00 \mathrm{E}+00$ & $0.00 \mathrm{E}+00$ & $1.54 \mathrm{E}+04$ \\
\hline climate change biogenic & 4.47E-03 & 4.06E-01 & $8.71 \mathrm{E}-03$ & $0.00 \mathrm{E}+00$ & 3.22E-02 & $1.40 \mathrm{E}-03$ & $0.00 \mathrm{E}+00$ & $0.00 \mathrm{E}+00$ & $0.00 \mathrm{E}+00$ & $1.46 \mathrm{E}+04$ \\
\hline freshwater \& terrestrial acidification & 8.85E-02 & $2.54 \mathrm{E}+00$ & $6.72 \mathrm{E}-02$ & $0.00 \mathrm{E}+00$ & 3.09E-01 & 5.09E-03 & $0.00 \mathrm{E}+00$ & $0.00 \mathrm{E}+00$ & $0.00 \mathrm{E}+00$ & $9.13 \mathrm{E}-01$ \\
\hline freshwater eutrophication & $5.49 \mathrm{E}-04$ & $6.58 \mathrm{E}-01$ & $6.54 \mathrm{E}-04$ & $0.00 \mathrm{E}+00$ & $7.80 \mathrm{E}-03$ & $9.40 \mathrm{E}-04$ & $0.00 \mathrm{E}+00$ & $0.00 \mathrm{E}+00$ & $0.00 \mathrm{E}+00$ & 8.11E-01 \\
\hline terrestrial eutrophication & 1.19E-01 & $3.86 \mathrm{E}+00$ & $1.72 \mathrm{E}-01$ & $0.00 \mathrm{E}+00$ & 7.52E-01 & $8.80 \mathrm{E}-03$ & $0.00 \mathrm{E}+00$ & $0.00 \mathrm{E}+00$ & $0.00 \mathrm{E}+00$ & $2.52 \mathrm{E}+00$ \\
\hline freshwater ecotoxicity & $8.03 \mathrm{E}+00$ & $2.19 \mathrm{E}+02$ & $8.54 \mathrm{E}+00$ & $0.00 \mathrm{E}+00$ & $3.23 \mathrm{E}+02$ & 7.47E-01 & $0.00 \mathrm{E}+00$ & $0.00 \mathrm{E}+00$ & $0.00 \mathrm{E}+00$ & $2.33 \mathrm{E}+03$ \\
\hline marine eutrophication & $1.09 \mathrm{E}-02$ & $4.80 \mathrm{E}-01$ & $1.59 \mathrm{E}-02$ & $0.00 \mathrm{E}+00$ & $6.92 \mathrm{E}-02$ & $9.89 \mathrm{E}-04$ & $0.00 \mathrm{E}+00$ & $0.00 \mathrm{E}+00$ & $0.00 \mathrm{E}+00$ & $1.13 \mathrm{E}+01$ \\
\hline ozone layer depletion & $1.13 \mathrm{E}-05$ & $5.01 \mathrm{E}-05$ & $4.24 \mathrm{E}-06$ & $0.00 \mathrm{E}+00$ & $2.22 \mathrm{E}-05$ & $5.21 \mathrm{E}-07$ & $0.00 \mathrm{E}+00$ & $0.00 \mathrm{E}+00$ & $0.00 \mathrm{E}+00$ & 1.05E-05 \\
\hline ionizing radiation & $3.11 \mathrm{E}+00$ & $2.51 \mathrm{E}+02$ & 1.33E-01 & $0.00 \mathrm{E}+00$ & $7.14 \mathrm{E}+00$ & 3.30E-01 & $0.00 \mathrm{E}+00$ & $0.00 \mathrm{E}+00$ & $0.00 \mathrm{E}+00$ & $3.06 \mathrm{E}+00$ \\
\hline photochemical ozone creation & $4.81 \mathrm{E}-02$ & $1.11 \mathrm{E}+00$ & $6.43 \mathrm{E}-02$ & $0.00 \mathrm{E}+00$ & 2.88E-01 & $2.52 \mathrm{E}-03$ & $0.00 \mathrm{E}+00$ & $0.00 \mathrm{E}+00$ & $0.00 \mathrm{E}+00$ & $5.23 \mathrm{E}+00$ \\
\hline carcinogenic effects & $5.74 \mathrm{E}-08$ & 4.83E-06 & $9.99 \mathrm{E}-08$ & $0.00 \mathrm{E}+00$ & $6.98 \mathrm{E}-07$ & $2.44 \mathrm{E}-08$ & $0.00 \mathrm{E}+00$ & $0.00 \mathrm{E}+00$ & $0.00 \mathrm{E}+00$ & 4.00E-05 \\
\hline non-carcinogenic effects & $5.71 \mathrm{E}-07$ & 4.62E-05 & $6.50 \mathrm{E}-07$ & $0.00 \mathrm{E}+00$ & $1.79 \mathrm{E}-05$ & $1.40 \mathrm{E}-07$ & $0.00 \mathrm{E}+00$ & $0.00 \mathrm{E}+00$ & $0.00 \mathrm{E}+00$ & $6.89 \mathrm{E}-05$ \\
\hline respiratory effects, inorganics & 4.92E-07 & $1.26 \mathrm{E}-05$ & $1.92 \mathrm{E}-07$ & $0.00 \mathrm{E}+00$ & $8.10 \mathrm{E}-06$ & 3.93E-08 & $0.00 \mathrm{E}+00$ & $0.00 \mathrm{E}+00$ & $0.00 \mathrm{E}+00$ & $1.38 \mathrm{E}-05$ \\
\hline minerals and metals & 3.73E-05 & $1.71 \mathrm{E}-03$ & $3.04 \mathrm{E}-05$ & $0.00 \mathrm{E}+00$ & $1.52 \mathrm{E}-03$ & $1.93 \mathrm{E}-05$ & $0.00 \mathrm{E}+00$ & $0.00 \mathrm{E}+00$ & $0.00 \mathrm{E}+00$ & 8.23E-05 \\
\hline fossils & $6.85 \mathrm{E}+02$ & $1.40 \mathrm{E}+04$ & $6.69 \mathrm{E}+02$ & $0.00 \mathrm{E}+00$ & $1.51 \mathrm{E}+03$ & $2.15 \mathrm{E}+01$ & $0.00 \mathrm{E}+00$ & $0.00 \mathrm{E}+00$ & $0.00 \mathrm{E}+00$ & $6.66 \mathrm{E}+02$ \\
\hline dissipated water & $9.39 \mathrm{E}-01$ & $1.56 \mathrm{E}+02$ & $2.58 \mathrm{E}+00$ & $0.00 \mathrm{E}+00$ & $6.76 \mathrm{E}+00$ & $2.07 \mathrm{E}+00$ & $0.00 \mathrm{E}+00$ & $0.00 \mathrm{E}+00$ & $0.00 \mathrm{E}+00$ & $1.78 \mathrm{E}+00$ \\
\hline land use & $8.11 \mathrm{E}+01$ & $1.53 \mathrm{E}+04$ & $7.11 \mathrm{E}+01$ & $0.00 \mathrm{E}+00$ & $3.55 \mathrm{E}+03$ & $3.65 E+01$ & $0.00 \mathrm{E}+00$ & $0.00 \mathrm{E}+00$ & $0.00 \mathrm{E}+00$ & $4.24 \mathrm{E}+03$ \\
\hline
\end{tabular}


Table S22. ILCD, 2.0 results for incineration of 1 ton of waste PP.

\begin{tabular}{|c|c|c|c|c|c|c|c|c|c|c|}
\hline Impact category & diesel & electricity & heat & petrol & transport & water & $\begin{array}{l}\text { organic } \\
\text { solvent }\end{array}$ & hydrogen & steam & $\begin{array}{c}\text { incineration } \\
\text { activities }\end{array}$ \\
\hline climate change fossil & 3.07E-01 & $6.09 \mathrm{E}+02$ & $6.53 \mathrm{E}-01$ & $0.00 \mathrm{E}+00$ & $2.00 \mathrm{E}+00$ & 4.07E-01 & $0.00 \mathrm{E}+00$ & $0.00 \mathrm{E}+00$ & $0.00 \mathrm{E}+00$ & $1.53 \mathrm{E}+01$ \\
\hline climate change land use & $1.50 \mathrm{E}+01$ & $1.52 \mathrm{E}+03$ & $2.13 \mathrm{E}+02$ & $0.00 \mathrm{E}+00$ & $9.41 \mathrm{E}+01$ & $1.28 \mathrm{E}+00$ & $0.00 \mathrm{E}+00$ & $0.00 \mathrm{E}+00$ & $0.00 \mathrm{E}+00$ & $5.74 \mathrm{E}+03$ \\
\hline climate change total & 4.22E-03 & 8.70E-01 & 1.10E-02 & $0.00 \mathrm{E}+00$ & 3.22E-02 & 1.15E-03 & $0.00 \mathrm{E}+00$ & $0.00 \mathrm{E}+00$ & $0.00 \mathrm{E}+00$ & 1.63E-01 \\
\hline climate change biogenic & $1.50 \mathrm{E}+01$ & $1.53 \mathrm{E}+03$ & $2.13 \mathrm{E}+02$ & $0.00 \mathrm{E}+00$ & $9.41 \mathrm{E}+01$ & $1.28 \mathrm{E}+00$ & $0.00 \mathrm{E}+00$ & $0.00 \mathrm{E}+00$ & $0.00 \mathrm{E}+00$ & $5.74 \mathrm{E}+03$ \\
\hline freshwater \& terrestrial acidification & 8.93E-03 & 8.12E-01 & 4.35E-02 & $0.00 \mathrm{E}+00$ & $3.22 \mathrm{E}-02$ & $1.40 \mathrm{E}-03$ & $0.00 \mathrm{E}+00$ & $0.00 \mathrm{E}+00$ & $0.00 \mathrm{E}+00$ & 4.55E-01 \\
\hline freshwater eutrophication & $1.77 \mathrm{E}-01$ & $5.08 \mathrm{E}+00$ & 3.36E-01 & $0.00 \mathrm{E}+00$ & 3.09E-01 & 5.09E-03 & $0.00 \mathrm{E}+00$ & $0.00 \mathrm{E}+00$ & $0.00 \mathrm{E}+00$ & $3.47 \mathrm{E}+00$ \\
\hline terrestrial eutrophication & $1.10 \mathrm{E}-03$ & $1.32 \mathrm{E}+00$ & 3.27E-03 & $0.00 \mathrm{E}+00$ & $7.80 \mathrm{E}-03$ & $9.40 \mathrm{E}-04$ & $0.00 \mathrm{E}+00$ & $0.00 \mathrm{E}+00$ & $0.00 \mathrm{E}+00$ & 4.37E-01 \\
\hline freshwater ecotoxicity & 2.37E-01 & $7.72 \mathrm{E}+00$ & 8.58E-01 & $0.00 \mathrm{E}+00$ & 7.52E-01 & $8.80 \mathrm{E}-03$ & $0.00 \mathrm{E}+00$ & $0.00 \mathrm{E}+00$ & $0.00 \mathrm{E}+00$ & $1.52 \mathrm{E}+01$ \\
\hline marine eutrophication & $1.61 \mathrm{E}+01$ & $4.39 \mathrm{E}+02$ & $4.27 \mathrm{E}+01$ & $0.00 \mathrm{E}+00$ & $3.23 \mathrm{E}+02$ & 7.47E-01 & $0.00 \mathrm{E}+00$ & $0.00 \mathrm{E}+00$ & $0.00 \mathrm{E}+00$ & $2.57 \mathrm{E}+04$ \\
\hline ozone layer depletion & $2.17 \mathrm{E}-02$ & $9.60 \mathrm{E}-01$ & 7.94E-02 & $0.00 \mathrm{E}+00$ & $6.92 \mathrm{E}-02$ & $9.89 \mathrm{E}-04$ & $0.00 \mathrm{E}+00$ & $0.00 \mathrm{E}+00$ & $0.00 \mathrm{E}+00$ & $1.78 \mathrm{E}+00$ \\
\hline ionizing radiation & $2.26 \mathrm{E}-05$ & $1.00 \mathrm{E}-04$ & $2.12 \mathrm{E}-05$ & $0.00 \mathrm{E}+00$ & $2.22 \mathrm{E}-05$ & $5.21 \mathrm{E}-07$ & $0.00 \mathrm{E}+00$ & $0.00 \mathrm{E}+00$ & $0.00 \mathrm{E}+00$ & 4.67E-05 \\
\hline photochemical ozone creation & $6.22 \mathrm{E}+00$ & $5.02 \mathrm{E}+02$ & $6.64 \mathrm{E}-01$ & $0.00 \mathrm{E}+00$ & $7.14 \mathrm{E}+00$ & 3.30E-01 & $0.00 \mathrm{E}+00$ & $0.00 \mathrm{E}+00$ & $0.00 \mathrm{E}+00$ & $1.63 \mathrm{E}+01$ \\
\hline carcinogenic effects & $9.62 \mathrm{E}-02$ & $2.23 \mathrm{E}+00$ & 3.22E-01 & $0.00 \mathrm{E}+00$ & $2.88 \mathrm{E}-01$ & $2.52 \mathrm{E}-03$ & $0.00 \mathrm{E}+00$ & $0.00 \mathrm{E}+00$ & $0.00 \mathrm{E}+00$ & $3.76 \mathrm{E}+00$ \\
\hline non-carcinogenic effects & $1.15 \mathrm{E}-07$ & $9.65 \mathrm{E}-06$ & 4.99E-07 & $0.00 \mathrm{E}+00$ & $6.98 \mathrm{E}-07$ & $2.44 \mathrm{E}-08$ & $0.00 \mathrm{E}+00$ & $0.00 \mathrm{E}+00$ & $0.00 \mathrm{E}+00$ & $9.65 \mathrm{E}-05$ \\
\hline respiratory effects, inorganics & $1.14 \mathrm{E}-06$ & $9.24 \mathrm{E}-05$ & $3.25 \mathrm{E}-06$ & $0.00 \mathrm{E}+00$ & $1.79 \mathrm{E}-05$ & $1.40 \mathrm{E}-07$ & $0.00 \mathrm{E}+00$ & $0.00 \mathrm{E}+00$ & $0.00 \mathrm{E}+00$ & 5.89E-04 \\
\hline minerals and metals & $9.83 \mathrm{E}-07$ & 2.53E-05 & $9.58 \mathrm{E}-07$ & $0.00 \mathrm{E}+00$ & $8.10 \mathrm{E}-06$ & 3.93E-08 & $0.00 \mathrm{E}+00$ & $0.00 \mathrm{E}+00$ & $0.00 \mathrm{E}+00$ & 2.92E-05 \\
\hline fossils & 7.46E-05 & $3.41 \mathrm{E}-03$ & $1.52 \mathrm{E}-04$ & $0.00 \mathrm{E}+00$ & $1.52 \mathrm{E}-03$ & 1.93E-05 & $0.00 \mathrm{E}+00$ & $0.00 \mathrm{E}+00$ & $0.00 \mathrm{E}+00$ & 4.23E-02 \\
\hline dissipated water & $1.37 \mathrm{E}+03$ & $2.80 \mathrm{E}+04$ & $3.35 \mathrm{E}+03$ & $0.00 \mathrm{E}+00$ & $1.51 \mathrm{E}+03$ & $2.15 \mathrm{E}+01$ & $0.00 \mathrm{E}+00$ & $0.00 \mathrm{E}+00$ & $0.00 \mathrm{E}+00$ & $3.87 \mathrm{E}+03$ \\
\hline land use & $1.88 \mathrm{E}+00$ & $3.11 \mathrm{E}+02$ & $1.29 \mathrm{E}+01$ & $0.00 \mathrm{E}+00$ & $6.76 \mathrm{E}+00$ & $2.07 \mathrm{E}+00$ & $0.00 \mathrm{E}+00$ & $0.00 \mathrm{E}+00$ & $0.00 \mathrm{E}+00$ & $7.71 \mathrm{E}+02$ \\
\hline
\end{tabular}


Table S23. Characterization factor units used for the ReCiPe method. ${ }^{28}$

\begin{tabular}{|c|c|c|}
\hline Sr. No. & Midpoint indicator & Impact score unit \\
\hline 1 & agricultural land occupation & $m^{2} a$ \\
\hline 2 & climate change & $\mathrm{kg} \mathrm{CO}_{2}$-eq \\
\hline 3 & fossil depletion & kg oil-eq \\
\hline 4 & freshwater ecotoxicity & kg 1,4-DC-eq \\
\hline 5 & freshwater eutrophication & kg P-Eq \\
\hline 6 & human toxicity & kg 1,4-DC-eq \\
\hline 7 & ionizing radiation & $\mathrm{kg} \mathrm{U}^{235}$-eq \\
\hline 8 & marine ecotoxicity & kg 1,4-DB-eq \\
\hline 9 & marine eutrophication & kg N-eq \\
\hline 10 & metal depletion & kg Fe-eq \\
\hline 11 & natural land transformation & $\mathrm{m}^{2}$ \\
\hline 12 & ozone depletion & kg CFC-11-eq \\
\hline 13 & particulate matter formation & kg $\mathrm{PM}^{10}$-eq \\
\hline 14 & photochemical oxidant formation & kg NMVOC-eq \\
\hline 15 & terrestrial acidification & $\mathrm{kg} \mathrm{SO}^{2}$-eq \\
\hline 16 & terrestrial ecotoxicity & kg 1,4-DC-eq \\
\hline 17 & urban land occupation & $\mathrm{m}^{2} \mathrm{a}$ \\
\hline 18 & water depletion & $\mathrm{m}^{3}$ water-eq \\
\hline
\end{tabular}

Table S24. Characterization factor units for the ILCD 2.0 method. ${ }^{29}$

\begin{tabular}{cll}
\hline Sr. No. & Midpoint indicator & Impact score unit \\
\hline 1 & climate change fossil & $\mathrm{kg} \mathrm{CO}_{2}$-eq \\
2 & climate change land use and land use change & $\mathrm{kg} \mathrm{CO}_{2}$-eq \\
3 & climate change total & $\mathrm{kg} \mathrm{CO}_{2}$-eq \\
4 & climate change biogenic & $\mathrm{kg} \mathrm{CO}_{2}$-eq \\
5 & freshwater and terrestrial acidification & $\mathrm{mol} \mathrm{H}$-eq \\
6 & terrestrial eutrophication & $\mathrm{mol} \mathrm{N}$-eq \\
7 & freshwater ecotoxicity & $\mathrm{CTUe}$ \\
8 & marine eutrophication & $\mathrm{kg} \mathrm{N}-\mathrm{Eq}$ \\
9 & freshwater eutrophication & $\mathrm{kg} \mathrm{P-Eq}$ \\
10 & photochemical ozone creation & $\mathrm{kg} \mathrm{NMVOC-eq}$ \\
11 & ozone layer depletion & $\mathrm{kg} \mathrm{CFC-11-eq}$ \\
12 & respiratory effects, inorganics & $\mathrm{kg} \mathrm{PM2.5} \mathrm{eq.}$ \\
13 & non-carcinogenic effects & $\mathrm{CTUh}$ \\
14 & carcinogenic effects & $\mathrm{CTUh}$
\end{tabular}


15 ionizing radiation

16 land use

17 fossils

18 minerals and metals

19 dissipated water
$\mathrm{kBq} \mathrm{U}^{235}$ eq. (to air)

points

MJ

$\mathrm{kg}$ Sb-eq

$\mathrm{m}^{3}$ water-eq

\section{S3. Pyrolysis of polypropylene mixtures}

\section{S3.1 Polypropylene interactions with polystyrene in pyrolysis}

No stream of plastic waste is uncontaminated. Thus, the literature was reviewed for PP interactions with polystyrene (PS), polyethylene (PE), polyethylene terephthalate (PET), and polyvinyl chloride (PVC) in pyrolysis. Wong and Broadbelt studied the pyrolysis of PP and PS mixtures, and found that the presence of PP did not affect PS degradation, but the presence of PS increased PP degradation. ${ }^{30}$ To explain this result, the authors proposed that styrene radicals formed as PS decomposed at lower temperatures helped initiate PP decomposition. This hypothesis was modelled in their subsequent paper, and their model successfully predicted the low molecular weight product (LMWP) yield as a function of time, supporting their hypothesis. Williams and Williams also studied the pyrolysis of PP + PS mixtures, and similarly found evidence of a synergistic effect. ${ }^{31}$

\section{S3.2 Polypropylene interactions with polyethylene in pyrolysis}

The literature reviewed presents mixed results regarding uncatalyzed PP and PE interactions in pyrolysis. Chowlu et al reports a decrease in decomposition temperature for LDPEPP mixture compared to the decomposition temperatures of the components, suggesting that LDPE-PP mixture degrades more readily than either of the components. ${ }^{7}$ Miandad et al, on the other hand, reports that pyrolysis of PP and PE (author did not distinguish between HDPE and LDPE) together produces much more char and less oil than either PP or PE individually. ${ }^{20}$ The extra char production disappears, however, when PS is pyrolyzed together with the mixture of PP and PE. In addition, Marcilla et al compared TGA curves of HDPE/PP mixtures with the curves of HDPE and PP individually, finding that the decomposition curves of the plastic mixtures are approximately linear combinations of the curves for individual plastics, suggesting the absence of any synergistic effect between PP and HDPE. ${ }^{32}$ 


\section{S3.3 Polypropylene interactions with polyvinyl chloride in pyrolysis}

It is well known that PVC contamination is problematic in thermochemical treatment of wastes - the chlorine content in PVC becomes hot, corrosive $\mathrm{HCl}$ gas in the reactor while producing chloro-organic compounds in the oil fraction of the product. ${ }^{33-35}$ Lopez et al investigated stepwise heating and $\mathrm{CaCO}_{3}$ addition as potential solutions to PVC contamination and found that stepwise heating reduced the chlorine content of product oils by $70 \%$, while $\mathrm{CaCO}_{3}$ decreased $\mathrm{HCl}$ release but increased chlorine content of the char. ${ }^{36}$ The stepwise heating method appears promising because $\mathrm{HCl}$ release occurs between 250 and $360^{\circ} \mathrm{C}$, where little if any PP degradation occurs. However, despite the TGA curve indicating nearly complete $\mathrm{HCl}$ release from PVC over this temperature range, $\mathrm{HCl}$ release has been found to be retarded when PVC is heating in a plastic mixture; the precise amount of $\mathrm{HCl}$ released in a preheating step varies from author to author, usually between 70 and $90 \%$. A third, simpler method for treating PVC contamination is through flotation separation - PVC sinks in water, while PP floats. This method brings the added benefit of a washing step for any contaminants, but would require extra energy input to dry the plastic feedstock before processing and may not separate PVC from any layered films. Overall, treating PVC's chlorine content in the plastics stream remains problematic.

\section{S3.4 Polypropylene interactions with polyethylene terephthalate in pyrolysis}

Like PVC, PET contamination is problematic in pyrolysis because of its elemental composition: PET's oxygen atoms cause partial oxidation of the products. Honus et al pyrolyzed various plastic mixtures and found product $\mathrm{CO}$ and $\mathrm{CO}_{2}$ for mixtures containing PET. In addition, the $\mathrm{CO}$ and $\mathrm{CO}_{2}$ do not account for all of the oxygen content in the PET-containing mixtures, indicating the presence of oxygen atoms in the liquid and solid fractions. This partial oxidation of the products means lower energy density within the products, which poses obvious problems if the products are to be used as fuels. Like PVC, however, PET sinks in water, so flotation separation may be feasible for treating PET contamination.

\section{S4. Gasification of polypropylene mixtures}

\section{S4.1 Polypropylene interactions with polyethylene in gasification}


As mentioned previously, no stream of plastic waste will ever be free of contamination. Thus, the literature was reviewed for interactions between different plastic resins in gasification. Wilk and Hofbauer investigated dual-chamber steam gasification of PP and PE mixtures, and found that the gasification of PP and PE together produced much less char and more product gas than either PP or PE separately, indicating a synergistic effect between PP and PE in steam gasification. ${ }^{37}$ In addition, Saebea et al simulated the steam gasification of PP and PE mixtures, finding that molar flow rate of syngas increased linearly (but only slightly) as a function of proportion of PE in the feedstock. ${ }^{38}$ These results together suggest that PE contamination poses few problems in PP stream gasification.

\section{S4.2 Polypropylene interactions with polystyrene in gasification}

Unfortunately, no literature could be found on the gasification of PP and PS mixture. The next closest analyses were Wu and Williams investigation of catalytic pyrolysis- steam gasification of PP, HDPE, and PS mixture (finding minimal evidence of interaction) and Janajreh et al. investigation of uncatalyzed air gasification PP, PE, and PS mixture (finding improved yields of the mixture compared to gasification of the individual components). ${ }^{39-40}$ These results together suggest that PS presence is beneficial to the gasification process when both PP and PE are present, however, these data do not show whether a similarly beneficial effect would be present if the mixture consisted of only PP and PS.

\section{S4.3 Polypropylene interactions with polyethylene terephthalate in gasification}

Unfortunately, no literature could be found on the interactions between PP and PET in gasification. The next closest analysis that could be found was by the simulations by Kannan et al. for co-gasification of PE with PET in steam and air. ${ }^{41}$ Like in pyrolysis, increasing PET content corresponded with a decrease in energy density of the product syngas, however, PET content was also correlated with an increase in gasification conversion efficiency and an increase in cold gas efficiency. Assuming that PP/PET mixture behaves similarly as PE/PET mixture, these results were interpreted to mean that PET contamination would have much of the same effect as increasing the ratio of oxidizing agent to input feed - decreased energy density of output but increased conversion. This suggests that PET in the waste stream can partially replace the gasifying agent as an oxidizer, however, further research is necessary to determine the feasibility of such an approach. 


\section{S4.4 Polypropylene interactions with polyvinyl chloride in gasification}

It is well established that PVC will release $\mathrm{HCl}$ upon gasification. Thus, PVC contamination poses similar problems in gasification as it does in pyrolysis. When PVC content in the input feedstock is high, the $\mathrm{HCl}$ can potentially be captured and sold as an additional product. ${ }^{42}$ When chlorine content in the feedstock is lower, however, $\mathrm{HCl}$ capture may become less feasible and neutralization strategies may become necessary, namely, additives either in the primary reactor or a downstream reactor to absorb the $\mathrm{HCl}$ as soon as it forms. Calcium, magnesium, and sodium salts, as well as natural minerals such as dolomite and oyster shells, have been studied as potential $\mathrm{HCl}$ adsorbents. ${ }^{3,43}$ Corella et al tested the efficacy of dolomite and quicklime $(\mathrm{CaO})$ in $\operatorname{tar}$ elimination and $\mathrm{HCl}$ adsorption, finding their utility limited because the product $\mathrm{CaCl}_{2}$ and $\mathrm{MgCl}_{2}$ tends to melt, agglomerate, and cause internal collapse of the apparatus. ${ }^{44}$ Borgianni et al compared the performance of $\mathrm{CaO}, \mathrm{Ca}(\mathrm{OH})_{2}$, and $\mathrm{Na}_{2} \mathrm{CO}_{3}$ in $\mathrm{HCl}$ adsorption, and also discovered the calcium salts to be inadequate at chlorine removal, but adding 1.2 times the stoichiometric amount of $\mathrm{Na}_{2} \mathrm{CO}_{3}$ led to over $99 \% \mathrm{HCl}$ cleansing from the product gas. ${ }^{43}$ Cho et al investigated oyster shells, $\mathrm{CaO}$, dolomite, activated carbon, and activated carbon with nickel for both simultaneous tar suppression and $\mathrm{HCl}$ suppression and removal, reporting that activated carbon, both with and without nickel, were the most effective at both tar suppression and $\mathrm{HCl}$ suppression, moving chlorine content from the gas product to the liquid condensate. ${ }^{3}$ Still, this research was conducted on either pure PVC streams or PVC mixtures with generic refuse derived fuels (RDFs), so more research is needed to determine whether these additives are equally as effective in treating PVC mixtures with PP and other plastics. Other methods of handling PVC contamination include stepwise heating and flotation separation, as discussed above. 


\section{References}

(1) Wu, C. F.; Williams, P. T., Hydrogen production by steam gasification of polypropylene with various nickel catalysts. Applied Catalysis B-Environmental 2009, 87 (3-4), 152-161, DOI: 10.1016/j.apcatb.2008.09.003.

(2) Xiao, R.; Jin, B. S.; Zhou, H. C.; Zhong, Z. P.; Zhang, M. Y., Air gasification of polypropylene plastic waste in fluidized bed gasifier. Energy Convers. Manage. 2007, 48 (3), 778-786, DOI: 10.1016/j.enconman.2006.09.004.

(3) Cho, M. H.; Choi, Y. K.; Kim, J. S., Air gasification of PVC (polyvinyl chloride)-containing plastic waste in a two-stage gasifier using Ca-based additives and Ni-loaded activated carbon for the production of clean and hydrogen-rich producer gas. Energy 2015, 87, 586-593, DOI: 10.1016/j.energy.2015.05.026.

(4) Arena, U.; Zaccariello, L.; Mastellone, M. L., Tar removal during the fluidized bed gasification of plastic waste. Waste Manage. 2009, 29 (2), 783-791, DOI: 10.1016/j.wasman.2008.05.010.

(5) Sancho, J. A.; Aznar, M. P.; Toledo, J. M., Catalytic air gasification of plastic waste (polypropylene) in fluidized bed. Part I: Use of in-gasifier bed additives. Ind. Eng. Chem. Res. 2008, 47 (4), 1005-1010, DOI: 10.1021/ie071023q.

(6) Kim, J. W.; Mun, T. Y.; Kim, J. O.; Kim, J. S., Air gasification of mixed plastic wastes using a two-stage gasifier for the production of producer gas with low tar and a high caloric value. Fuel 2011, 90 (6), 2266-2272, DOI: 10.1016/j.fuel.2011.02.021.

(7) Chowlu, A. C. K.; Reddy, P. K.; Ghoshal, A. K., Pyrolytic decomposition and model-free kinetics analysis of mixture of polypropylene (PP) and low-density polyethylene (LDPE). Thermochimica Acta 2009, 485 (1-2), 20-25, DOI: 10.1016/j.tca.2008.12.004.

(8) Lopez, G.; Artetxe, M.; Amutio, M.; Alvarez, J.; Bilbao, J.; Olazar, M., Recent advances in the gasification of waste plastics. A critical overview. Renewable Sustainable Energy Rev. 2018, 82, 576-596, DOI: 10.1016/j.rser.2017.09.032.

(9) Ciliz, N. K.; Ekinci, E.; Snape, C. E., Pyrolysis of virgin and waste polypropylene and its mixtures with waste polyethylene and polystyrene. Waste Manage. 2004, 24 (2), 173-181, DOI: 10.1016/j.wasman.2003.06.002.

(10) Predel, M.; Kaminsky, W., Pyrolysis of mixed polyolefins in a fluidised-bed reactor and on a pyro-GC/MS to yield aliphatic waxes. Polymer Degradation Stability 2000, 70 (3), 373-385, DOI: 10.1016/s0141-3910(00)00131-2.

(11) Williams, P. T.; Slaney, E., Analysis of products from the pyrolysis and liquefaction of single plastics and waste plastic mixtures. Resources Conserv. Recycling 2007, 51 (4), 754-769, DOI: 10.1016/j.resconrec.2006.12.002.

(12) Kruse, T. M.; Wong, H. W.; Broadbelt, L. J., Mechanistic modeling of polymer pyrolysis: Polypropylene. Macromolecules 2003, 36 (25), 9594-9607, DOI: 10.1021/ma030322y.

(13) Achilias, D. S.; Roupakias, C.; Megalokonomos, P.; Lappas, A. A.; Antonakou, E. V., Chemical recycling of plastic wastes made from polyethylene (LDPE and HDPE) and polypropylene (PP). J. Haz. Mat. 2007, 149 (3), 536-542, DOI: 10.1016/j.jhazmat.2007.06.076.

(14) Kassargy, C.; Awad, S.; Burnens, G.; Kahine, K.; Tazerout, M., Gasoline and diesel-like fuel production by continuous catalytic pyrolysis of waste polyethylene and polypropylene mixtures over USY zeolite. Fuel 2018, 224, 764-773, DOI: 10.1016/j.fuel.2018.03.113.

(15) Janajreh, I.; Adeyemi, I.; Elagroudy, S., Gasification feasibility of polyethylene, polypropylene, polystyrene waste and their mixture: Experimental studies and modeling. 
Sustainable Energy Technologies and Assessments 2020, 39, 100684, DOI: 10.1016/j.seta.2020.100684.

(16) Honus, S.; Kumagai, S.; Fedorko, G.; Molnar, V.; Yoshioka, T., Pyrolysis gases produced from individual and mixed PE, PP, PS, PVC, and PET-Part I: Production and physical properties. Fuel 2018, 221, 346-360, DOI: 10.1016/j.fuel.2018.02.074.

(17) Honus, S.; Kumagai, S.; Molnar, V.; Fedorko, G.; Yoshioka, T., Pyrolysis gases produced from individual and mixed PE, PP, PS, PVC, and PET-Part II: Fuel characteristics. Fuel 2018, 221, 361-373, DOI: 10.1016/j.fuel.2018.02.075.

(18) Feedstock Recycling and Pyrolysis of Waste Plastics: Converting Waste Plastics into Diesel and Other Fuels. Schiers, J.; Kaminsky, W., Eds. John Wiley \& Sons, Ltd: West Sussex, UK, 2006. (19) Gracida-Alvarez, U. R.; Winjobi, O.; Sacramento-Rivera, J. C.; Shonnard, D. R., System Analyses of High-Value Chemicals and Fuels from a Waste High-Density Polyethylene Refinery. Part 1: Conceptual Design and Techno-Economic Assessment. ACS Sustainable Chem. Eng. 2019, 7 (22), 18254-18266, DOI: 10.1021/acssuschemeng.9b04763.

(20) Miandad, R.; Barakat, M. A.; Aburiazaiza, A. S.; Rehan, M.; Ismail, I. M. I.; Nizami, A. S., Effect of plastic waste types on pyrolysis liquid oil. Int. Biodeterioration Biodegradation 2017, 119, 239-252, DOI: 10.1016/j.ibiod.2016.09.017.

(21) Swanson, R. M.; Platon, A.; Satrio, J. A.; Brown, R. C.; Hsu, D. D. Techno-Economic Analysis of Biofuels Production Based on Gasification; NREL/TP-6A20-46587; National Renewable Energy Lab. (NREL): Golden, CO, 2010.

(22) Wright, M. M.; Satrio, J. A.; Brown, R. C.; Daugaard, D. E.; Hsu, D. D. Techno-Economic Analysis of Biomass Fast Pyrolysis to Transportation Fuels; NREL/TP-6A20-46586; National Renewable Energy Lab. (NREL): Golden, CO (United States), 2010.

(23) Assamoi, B.; Lawryshyn, Y., The environmental comparison of landfilling vs. incineration of MSW accounting for waste diversion. Waste Manage. 2012, 32 (5), 1019-1030, DOI: 10.1016/j.wasman.2011.10.023.

(24) Bridgwater, A. V., Review of fast pyrolysis of biomass and product upgrading. Biomass Bioenergy 2012, 38, 68-94, DOI: 10.1016/j.biombioe.2011.01.048.

(25) Jones, S. B.; Zhu, Y.; Anderson, D. B.; Hallen, R. T.; Elliott, D. C.; Schmidt, A. J.; Albrecht, K. O.; Hart, T. R.; Butcher, M. G.; Drennan, C.; Snowden-Swan, L. J.; Davis, R.; Kinchin, C. Process Design and Economics for the Conversion of Algal Biomass to Hydrocarbons: Whole Algae Hydrothermal Liquefaction and Upgrading; PNNL-23227; Pacific Northwest National Lab. (PNNL): Richland, WA, 2014.

(26) Jones, S. B.; Valkenburt, C.; Walton, C. W.; Elliott, D. C.; Holladay, J. E.; Stevens, D. J.; Kinchin, C.; Czernik, S. Production of Gasoline and Diesel from Biomass via Fast Pyrolysis, Hydrotreating and Hydrocracking: A Design Case; PNNL-18284 Rev. 1; Pacific Northwest National Lab. (PNNL), Richland, WA (United States): 2009.

(27) Peters, M. S.; Timmerhaus, K. D.; West, R. E., Plant Design and Economics for Chemical Engineers. 5 ed.; McGraw-Hill Inc.: New York, NY, United States, 2003.

(28) Goedkoop, M.; Heijungs, R.; Huijbregts, M.; De Schryver, A.; Struijs, J.; Van Zelm, R. ReCiPe 2008: A life cycle impact assessment method which comprises harmonised category indicators at the midpoint and the endpoint level; Netherlands, 2009.

(29) Fazio, S.; Castellani, V.; Sala, S.; Schau, E.; Secchi, M.; Zampori, L.; Edward, D. Supporting information to the characterisation factors of recommended EF Life Cycle Impact Assessment methods; European Commission: Ispra, 2018. 
(30) Wong, H. W.; Broadbel, L. J., Tertiary resource recovery from waste polymers via pyrolysis: Neat and binary mixture reactions of polypropylene and polystyrene. Ind. Eng. Chem. Res. 2001, 40 (22), 4716-4723, DOI: 10.1021/ie010171s.

(31) Williams, P. T.; Williams, E. A., Interaction of plastics in mixed-plastics pyrolysis. Energy Fuels 1999, 13 (1), 188-196, DOI: 10.1021/ef980163x.

(32) Marcilla, A.; Garcia-Quesada, J. C.; Sanchez, S.; Ruiz Femenia, R., Study of the catalytic pyrolysis behaviour of polyethylene-polypropylene mixtures. J. Anal. Appl. Pyrolysis 2005, 74 (12), 387-392, DOI: 10.1016/j.jaap.2004.10.005.

(33) Miranda, R.; Pakdel, H.; Roy, C.; Vasile, C., Vacuum pyrolysis of commingled plastics containing PVC II. Product analysis. Polymer Degradation Stability 2001, 73 (1), 47-67, DOI: 10.1016/s0141-3910(01)00066-0.

(34) Miranda, R.; Yang, J.; Roy, C.; Vasile, C., Vacuum pyrolysis of commingled plastics containing PVC - I. Kinetic study. Polymer Degradation Stability 2001, 72 (3), 469-491, DOI: 10.1016/s0141-3910(01)00048-9.

(35) Yu, J.; Sun, L. S.; Ma, C.; Qiao, Y.; Yao, H., Thermal degradation of PVC: A review. Waste Manage. 2016, 48, 300-314, DOI: 10.1016/j.wasman.2015.11.041.

(36) Lopez-Urionabarrenechea, A.; de Marco, I.; Caballero, B. M.; Laresgoiti, M. F.; Adrados, A., Dechlorination of fuels in pyrolysis of PVC containing plastic wastes. Fuel Processing Technol. 2011, 92 (2), 253-260, DOI: 10.1016/j.fuproc.2010.05.008.

(37) Wilk, V.; Hofbauer, H., Conversion of mixed plastic wastes in a dual fluidized bed steam gasifier. Fuel 2013, 107, 787-799, DOI: 10.1016/j.fuel.2013.01.068.

(38) Saebea, D.; Ruengrit, P.; Arpornwichanop, A.; Patcharavorachot, Y., Gasification of plastic waste for synthesis gas production. Energy Reports 2020, 6, 202-207, DOI: 10.1016/j.egyr.2019.08.043.

(39) Janajreh, I.; Adeyemi, I.; Elagroudy, S., Gasification feasibility of polyethylene, polypropylene, polystyrene waste and their mixture: Experimental studies and modeling. Sustainable Energy Technol. Assess. 2020, 39, DOI: 10.1016/j.seta.2020.100684.

(40) Wu, C. F.; Williams, P. T., Pyrolysis-gasification of plastics, mixed plastics and real-world plastic waste with and without Ni-Mg-Al catalyst. Fuel 2010, 89 (10), 3022-3032, DOI: 10.1016/j.fuel.2010.05.032.

(41) Kannan, P.; Al Shoaibi, A.; Srinivasakannan, C., Energy recovery from co-gasification of waste polyethylene and polyethylene terephthalate blends. Comput. Fluids 2013, 88, 38-42, DOI: 10.1016/j.compfluid.2013.09.004.

(42) Slapak, M. J. P.; van Kasteren, J. M. N.; Drinkenburg, A. A. H., Design of a process for steam gasification of PVC waste. Resources Conserv. Recycling 2000, 30 (2), 81-93, DOI: 10.1016/s0921-3449(00)00047-1.

(43) Borgianni, C.; De Filippis, P.; Pochetti, F.; Paolucci, M., Gasification process of wastes containing PVC. Fuel 2002, 81 (14), 1827-1833, DOI: 10.1016/s0016-2361(02)00097-2.

(44) Corella, J.; Toledo, J. M.; Molina, G., Performance of $\mathrm{CaO}$ and $\mathrm{MgO}$ for the hot gas clean up in gasification of a chlorine-containing (RDF) feedstock. Bioresour. Technol. 2008, 99 (16), 7539-7544, DOI: 10.1016/j.biortech.2008.02.018. 